\title{
Enantioselective Synthesis of 2-Amino-1,1-diarylalkanes Bearing a Carbocyclic Ring Substituted Indole through Asymmetric Catalytic Reaction of Hydroxyindoles with Nitroalkenes
}

\author{
Carlos Vila, ${ }^{\dagger \dagger}$ Jaume Rostoll-Berenguer, ${ }^{\dagger, \S}$ Rubén Sánchez-García, ${ }^{\dagger} \S$ Gonzalo Blay, ${ }^{\dagger}$ \\ Isabel Fernández, M. Carmen Muñoz, and José R. Pedro* ${ }^{\dagger}{ }^{\dagger}$.
}

${ }^{\dagger}$ Departament de Química Orgànica, Facultat de Química, Universitat de València, Dr. Moliner 50, Burjassot, València 46100, Spain

${ }^{\ddagger}$ Departament de Física Aplicada, Universitat Politècnica de València, Camino de Vera s/n, València 46022, Spain

Supporting Information

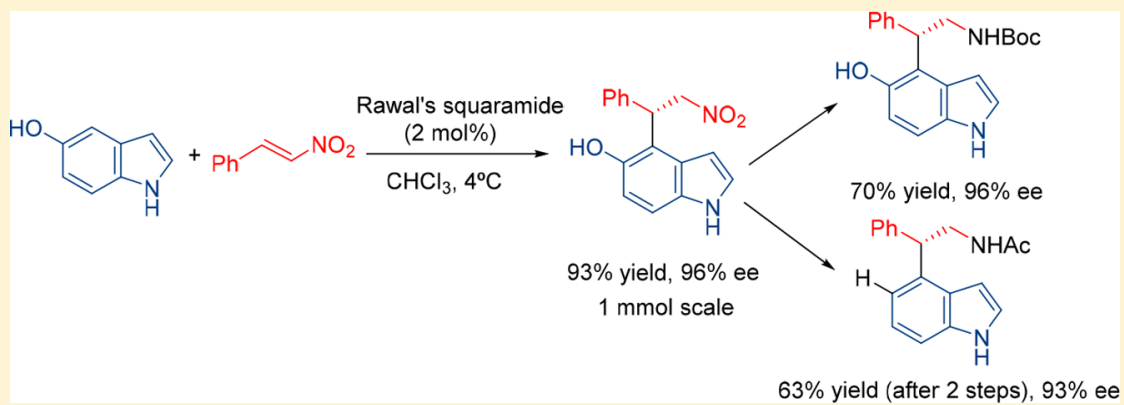

ABSTRACT: An asymmetric catalytic reaction of hydroxyindoles with nitroalkenes leading to the Friedel-Crafts alkylation in the carbocyclic ring of indole is presented. The method is based on the activating/directing effects of the hydroxy group situated in the carbocyclic ring of the indole providing nitroalkylated indoles functionalizated at the C-4, C-5, and C-7 positions with high yield, regio-, and enantioselectivity. The optically enriched nitroalkanes were transformed efficiently in optically enriched 2 amino-1,1-diarylalkanes bearing a carbocyclic ring substituted indole.

\section{INTRODUCTION}

The arylethylamine moiety is present as a basic skeleton of a series of compounds that exhibit significant pharmaceutical properties. $^{1}$ The installation of a second arene moiety at the benzylic position of this arylethylamine scaffold leads to a new structural motif, 2-amino-1,1-diarylalkane, with interesting biological functions (Figure 1). ${ }^{2}$ Among them, the heteroarylsubstituted derivatives represent an important class, particularly those incorporating an indole ${ }^{3}$ core because of the importance of this heterocyclic in medicinal ${ }^{4}$ and agrochemical chemistry ${ }^{5}$

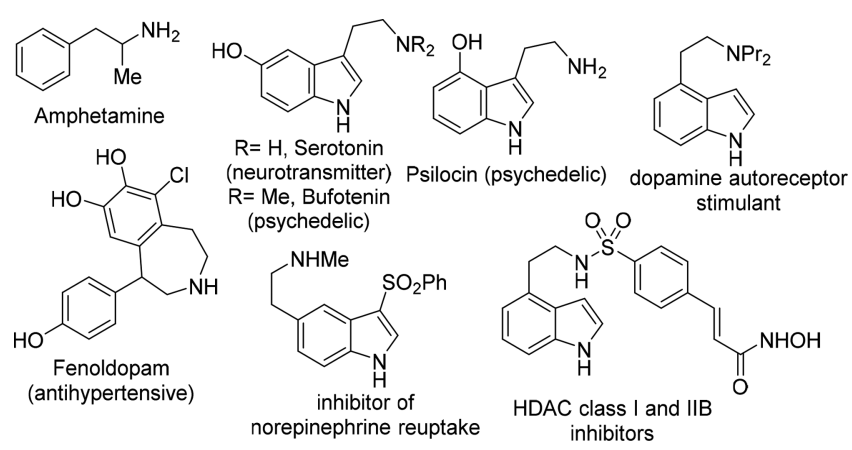

Figure 1. Examples of biologically active 2-amino-1-aryl alkanes, 2amino-1,1-diarylalkanes, and hydroxyindoles. and material science. ${ }^{6}$ Consequently, the enantioselective synthesis of this kind of compounds constitutes a major theme of recent research using asymmetric catalytic reactions (ACR).$^{7,8}$ Taking into consideration that nitroalkenes represent a notable class of electrophiles for $\mathrm{ACR},{ }^{9}$ because of the versatility of the resulting nitroalkanes as valuable intermediates in organic synthesis, ${ }^{10}$ we envisioned a procedure for the synthesis of 2-amino-1,1-diarylalkanes (Scheme 1). The procedure involves the introduction of an indole framework in the $\beta$-position of a nitrostyrene via a Friedel-Crafts alkylation followed by reduction of the nitro group to amine.

The majority of methods on the enantioselective FriedelCrafts reaction of indoles involve the azole ring. ${ }^{8}$ This fact can be explained by the inherent reactivity of indoles where the azole ring possesses a high nucleophilicity, the C-3 position being the most reactive. Additionally, different methodologies to perform the asymmetric functionalization at the C-2 position or the nitrogen atom have been described recently. ${ }^{11,12}$

In contrast, the functionalization of the carbocyclic ring is more challenging, and only some nonenantioselective methodologies have been described. ${ }^{13}$ However, the enantioselective functionalizations of positions C-4, C-5, C-6, and C-7 are

Received: March 7, 2018

Published: June 1, 2018 
Scheme 1. Enantioselective Functionalization of Indoles and Hydroxyindoles with $\boldsymbol{\alpha}, \boldsymbol{\beta}$-Unsaturated Nitroalkenes
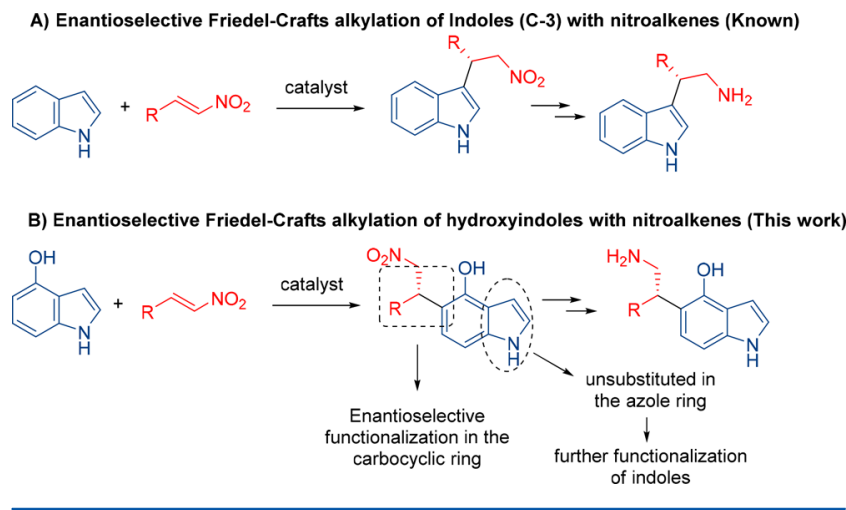

seldom studied in the literature. Therefore, the development of methodologies that enable the asymmetric functionalization of the carbocyclic ring of indoles is a great challenge in asymmetric catalysis. We have recently presented a methodology for the organocatalytic enantioselective functionalization of the carbocyclic ring of indoles using a hydroxy group as an activating/directing group. ${ }^{14-16}$ Hydroxindoles, using bifunctional organocatalysts, act as phenols, even when the positions in the azole ring of indole remain unsubstituted. Given the outstanding potential of chiral indoles and hydroxyindoles, the development of new methodologies for the enantioselective functionalization of indoles is of great interest.

In this Article, we described the enantioselective alkylation of hydroxyindoles with $\beta$-nitrostyrenes. Several enantioselective Friedel-Crafts reactions of indoles with nitroalkenes ${ }^{17,18}$ have been described in the literature for the synthesis of chiral triptamines (Scheme 1A). However, these examples are limited to the functionalization of the azole ring. The corresponding functionalization in the carbocyclic ring with nitroalkenes is unprecedented, to the best of our knowledge (Scheme 1B). The corresponding chiral nitroalkylated indoles in the carbocyclic ring are significant because of the possibility of reducing the nitro group and to synthesize 2-amino-1,1diarylalkanes.

\section{RESULTS AND DISCUSSION}

We started our studies with the Friedel-Crafts reaction of 4hydroxyindole (1a) with $\beta$-nitrostyrene (2a) in the presence of chiral bifunctional organocatalysts ${ }^{19}$ in toluene as a solvent at room temperature (Table 1 ). When quinine was used as a catalyst, full conversion was observed after $24 \mathrm{~h}$, and three products were identified in the crude reaction mixture: the corresponding alkylated products at C-5 position (3aa) and C7 position (3aa') and the dialkylated hydroxyindole at C-5 and C-7 positions (4), in a ratio 38:2:68 (3aa:3aa':4) respectively. We could isolate pure product 3 aa in $18 \%$ yield as a racemate (entry 1, Table 1). With catalyst II we could increase the yield and the ee of product 3aa (entry 2), but both still were very poor. When the thiourea derived from quinine III (entry 3 ) was used as catalyst, the reaction was more selective toward product 3aa, which was gained with $56 \%$ yield and $31 \%$ ee. The use of thiourea IV as catalyst (entry 4) gave worse results than did thiourea III. However, the squaramide V proved to be superior to the thiourea III, obtaining the best regioselectivity (63\% yield for 3aa) and enantioselectivity ( $83 \%$ ee, entry 5 ). While squaramide VI (entry 7 ), introduced by Rawal, ${ }^{20}$ gave the best
Table 1. Optimization of the Reaction Conditions

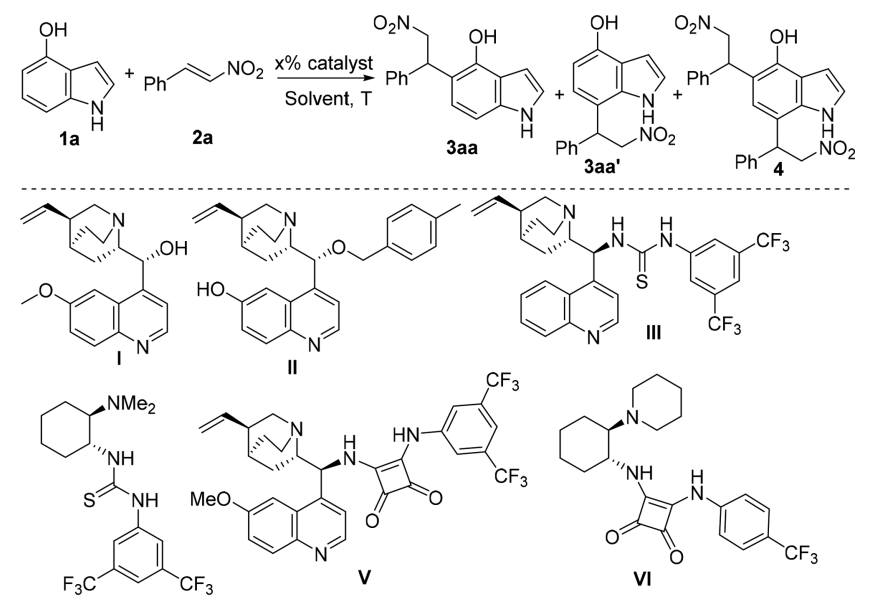

IV

\begin{tabular}{|c|c|c|c|c|c|c|}
\hline entry & catalyst & solvent & $\begin{array}{c}t \\
(\mathrm{~h})\end{array}$ & $\begin{array}{l}\text { percentage }^{d} \\
3 \text { aa:3aa':4 }\end{array}$ & $\begin{array}{c}\text { yield } \\
\text { of } 3 \mathbf{a a} \\
(\%)^{e}\end{array}$ & $\begin{array}{l}\text { ee of } \\
3 a a^{f}\end{array}$ \\
\hline $1^{a}$ & I $(10 \%)$ & toluene & 24 & $38: 2: 68$ & 18 & 2 \\
\hline $2^{a}$ & ${ }_{(10 \%)}^{\text {II }}$ & toluene & 23 & $53: 10: 37$ & 25 & 9 \\
\hline $3^{a}$ & ${ }_{(10 \%)}^{\text {III }}$ & toluene & 6 & $76: 2: 22$ & 56 & 31 \\
\hline $4^{a}$ & ${ }_{(10 \%)}^{\text {IV }}$ & toluene & 5 & $53: 2: 45$ & 43 & 3 \\
\hline $5^{a}$ & $\mathbf{V}_{(10 \%)}$ & toluene & 2 & $90: 2: 8$ & 63 & 83 \\
\hline $6^{a}$ & $\mathbf{V}(5 \%)$ & toluene & 7 & $92: 2: 6$ & 56 & 80 \\
\hline $7^{a, b}$ & $\begin{array}{l}\text { VI } \\
(5 \%)\end{array}$ & toluene & 24 & $86: 3: 10$ & 58 & 86 \\
\hline $8^{a, b}$ & $\begin{array}{l}\text { VI } \\
(5 \%)\end{array}$ & $\mathrm{CH}_{2} \mathrm{Cl}_{2}$ & 17 & $95: 0: 5$ & 71 & 91 \\
\hline $9^{a, b}$ & ${ }_{(5 \%)}$ & $\mathrm{ClCH}_{2} \mathrm{CH}_{2} \mathrm{Cl}$ & 17 & $93: 0: 7$ & 70 & 90 \\
\hline $10^{a, b}$ & $\begin{array}{l}\text { VI } \\
(5 \%)\end{array}$ & $\mathrm{CHCl}_{3}$ & 24 & $93: 1: 6$ & 86 & 93 \\
\hline $11^{a, b}$ & ${ }_{(5 \%)}^{\text {VI }}$ & MTBE & 48 & $55: 31: 14$ & 25 & n.d. ${ }^{g}$ \\
\hline $12^{b, c}$ & $\begin{array}{l}\text { VI } \\
(5 \%)\end{array}$ & $\mathrm{CHCl}_{3}$ & 8 & $98: 0: 2$ & 93 & 90 \\
\hline $13^{b, c}$ & ${ }_{(2 \%)}$ & $\mathrm{CHCl}_{3}$ & 22 & 100:0:0 & 80 & 92 \\
\hline $14^{b, c}$ & $\begin{array}{l}\text { VI } \\
(1 \%)\end{array}$ & $\mathrm{CHCl}_{3}$ & 22 & 100:0:0 & 70 & 90 \\
\hline
\end{tabular}

${ }^{a}$ Reaction conditions: $0.1 \mathrm{mmol}$ of $1 \mathrm{a}, 0.1 \mathrm{mmol} \mathrm{2a}, x \mathrm{~mol} \%$ de catalyst in $1 \mathrm{~mL}$ of solvent at rt. ${ }^{b}$ The temperature was $4{ }^{\circ} \mathrm{C}$. ${ }^{c}$ Reaction conditions: $0.15 \mathrm{mmol}$ of $1 \mathrm{a}, 0.1 \mathrm{mmol}$ of $2 \mathrm{a}, x \mathrm{~mol} \%$ of catalyst in 1 $\mathrm{mL}$ of solvent. ${ }^{d}$ Determined by ${ }^{1} \mathrm{H}$ NMR. ${ }^{e}$ Isolated yield of $3 a a$. $f_{\text {Determined by chiral HPLC. }}{ }^{g}$ Not determined.

enantiomeric excess ( $86 \%$ ee), and good yield (58\%), it was not sufficient for us. A solvent screening (entries 7-11) with catalyst VI showed that chlorinated solvents performed the reaction with the best enantioselectivities. Chloroform afforded the product 3aa with the best enantiomeric excess (93\% ee), and the best yield ( $86 \%$, entry 10$)$. To avoid the polyalkylated product 4 and increase the selectivity of product 3aa, we increased the ratio between 1a and 2a (entry 12), obtaining a slight decrease in the enantioselectivity. After, we screened the catalyst loading, and we found that we could decrease the catalyst loading to $2 \mathrm{~mol} \%$ (entry 13 ) without compromising the yield (80\%) and enantiomeric excess ( $92 \%$ ee). Furthermore, in these conditions, the dialkylation product 4 was not observed by ${ }^{1} \mathrm{H} \mathrm{NMR}$ in the reaction mixture. 
With the optimized reaction conditions established, the scope of the reaction was explored with respect to the 4hydroxyindole 1a and different $\beta$-nitrostyrenes $\mathbf{2}$ (Scheme 2). A

Scheme 2. Scope of the Friedel-Crafts Reaction with 4Hydroxyindole $1 \mathrm{a}^{a}$

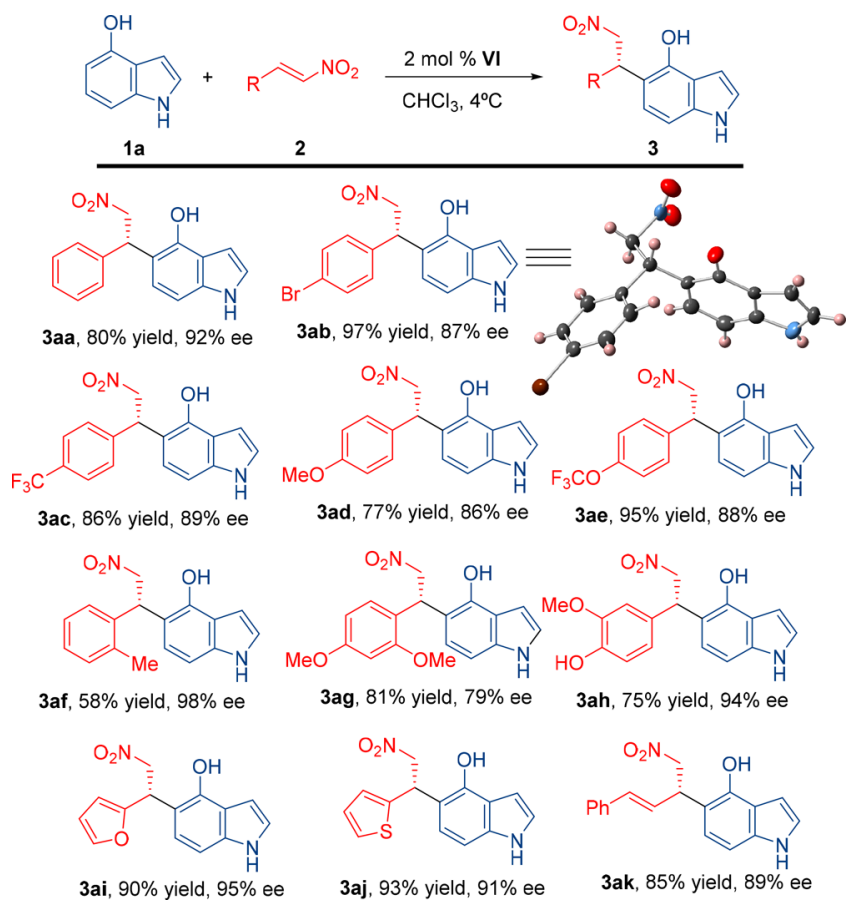

${ }^{a}$ Reaction conditions: 1a $(0.15 \mathrm{mmol}), 2(0.1 \mathrm{mmol})$, and VI $(2 \mathrm{~mol}$ $\%)$ in $1 \mathrm{~mL}$ of $\mathrm{CHCl}_{3}$ at $4{ }^{\circ} \mathrm{C}$. Isolated yields after column chromatography. Enantiomeric excesses were determined by HPLC using chiral stationary phase.

range of $\beta$-arylnitroalkenes were suitable for this reaction obtaining good yields and enantioselectivities. Electron-withdrawing $\left(\mathrm{Br}, \mathrm{CF}_{3}\right)$ and electron-donating groups $\left(\mathrm{MeO}, \mathrm{CF}_{3} \mathrm{O}\right)$ in para position were well tolerated. The presence of substituents at ortho position (Me) led to an excellent enantioselectivity (98\% ee), although with a decrease in the conversion ( $58 \%$ yield). When $\beta$-heteroarylnitroalkenes $2 \mathbf{i}$ and $2 \mathbf{j}$ were used, excellents yields and enantioselectivities were obtained. Remarkably, when (E)-2-methoxy-4-(2-nitrovinyl)phenol $\mathbf{2 h}$, with an hydroxyl group that may interfere in the catalysis, was used as an electrophile, the corresponding product 3ah was gained with excellent regio- and enantioselectivity (94\% ee). Finally, with $((1 E, 3 E)$-4-nitrobuta-1,3-dien1 -yl)benzene $2 \mathbf{k}$, product $3 \mathrm{ak}$ was obtained regioselectively with $85 \%$ yield and $89 \%$ ee. This result is particularly noteworthy, due to the possible regioisomers than can be obtained in this reaction. From the point of view of the nucleophile, hydroxyindole 1a can be alkylated at C-3, C-5, or C-7, but we only observe alkylation at C-5. From the point of view of the electrophile, we can have 1,4- or 1,6-addition, but we only observed the 1,4-addition product. The absolute configuration of product $3 \mathbf{a b}$ was determined by X-ray analysis, $^{21}$ and for the rest of compounds 3, we assumed a uniform stereochemical mechanism.

After having proved the efficiency of our methodology for the regio- and enantioselective Friedel-Crafts alkylation of 4hydroxyindole with nitroalkenes at the C-5 position, we examined the scope of the reaction with indoles bearing a hydroxy group in other positions of the carbocyclic ring. In this way, we wanted to explore if we could achieve the enantioselective functionalization of every position in this ring by simply changing the position of the directing group. Pleasingly, 5-hydroxyindole (1b) reacted with $\beta$-nitrostyrene (2a) to give regioselectivity C-4 alkylated product 3 ba with good yield (79\% yield) and high enantiocontrol (96\% ee) (Scheme 3 ). We carried out the reaction of $\mathbf{1} \mathbf{b}$ with several $\beta$ -

Scheme 3. Scope of the Friedel-Crafts Reaction with 5Hydroxyindole Derivatives ${ }^{a}$

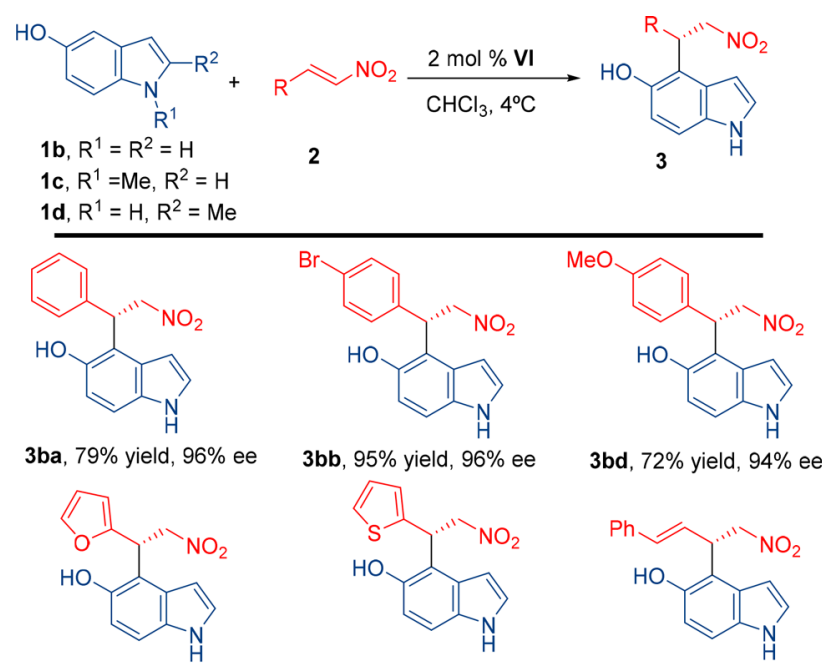

3 bi, $83 \%$ yield, $95 \%$ ee

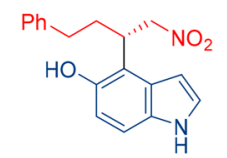

3 bl, ${ }^{\mathrm{b}} 44 \%$ yield, $87 \%$ ee

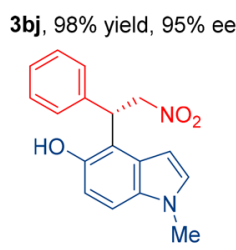

3ca, $88 \%$ yield, $96 \%$ ee

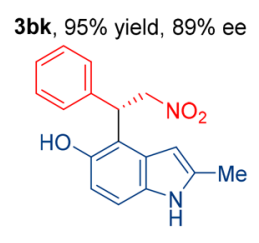

3da, $80 \%$ yield, $96 \%$ ee
${ }^{a}$ The reaction was performed at $50{ }^{\circ} \mathrm{C}$ using $5 \mathrm{~mol} \%$ of VI. Reaction conditions: $1(0.15 \mathrm{mmol}), 2(0.1 \mathrm{mmol})$, and VI $(2 \mathrm{~mol} \%)$ in $1 \mathrm{~mL}$ of $\mathrm{CHCl}_{3}$ at $4{ }^{\circ} \mathrm{C}$. Isolated yields after column chromatography. Enantiomeric excesses were determined by HPLC using chiral stationary phase.

aryl and $\beta$-heteroarylnitroalkenes obtaining high yields and better enantioselectivities (up to $96 \%$ ee) than the reactions with 1a. Moreover, the reaction with ((1E,3E)-4-nitrobuta-1,3dien-1-yl)benzene $\mathbf{2} \mathbf{k}$ procceded with excellent regioselectivity (only one product was observed) and high enantioselectivity, and product 3bk was gained with $95 \%$ yield and $89 \%$ ee. Furthermore, the reaction was carried out with one $\beta$ alkylnitroalkene. In this case, the reactivity was very low, and we needed to increase the temperature to $50{ }^{\circ} \mathrm{C}$ and the catalyst loading to $5 \mathrm{~mol} \%$ for several days. In these conditions, the reaction of $\mathbf{1} \mathbf{b}$ with $(E)$-(4-nitrobut-3-en-1-yl)benzene gave regioselectively compound $3 \mathrm{bl}$ with moderate $44 \%$ yield, but good enantioselectivity ( $87 \%$ ee). 5-Hydroxyindoles with alkyl substituents at the pyrrole ring were also tested, and products $3 \mathrm{ca}$ and $3 \mathrm{da}$ were isolated in high yields with excellent enantioselectivities (96\% ee). In the reaction with 5-hydroxy-2methyl-indole 1d, we also observed the C-3 alkylated product ( $15 \%$ yield) as a racemic mixture.

We also extend our methodology to 6-hydroxyindole 1e (Scheme 4), which was regio- and enantioselectively alkylated at the C-7 position with good yields and excellent enantiomeric 
Scheme 4. Scope of the Friedel-Crafts Reaction with 6Hydroxyindole $1 \mathrm{e}^{a}$

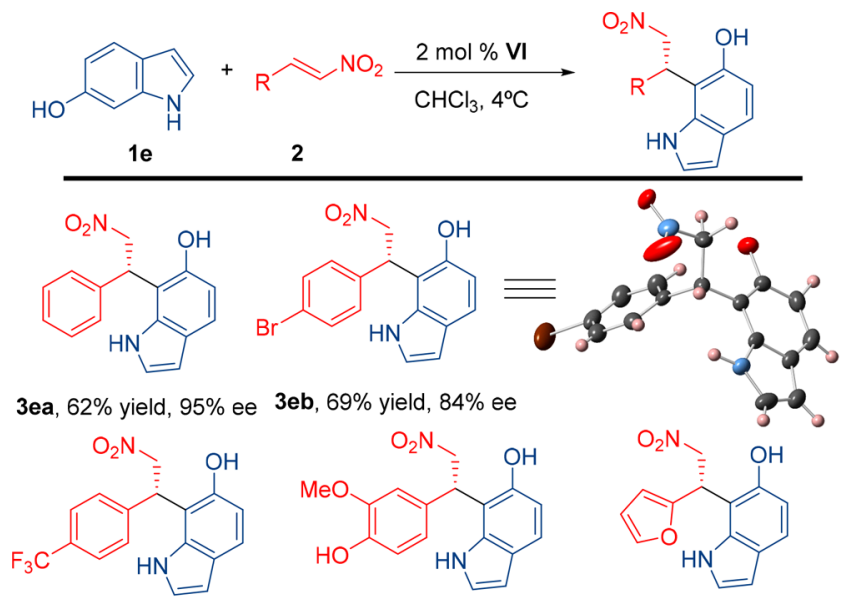

3ec, $77 \%$ yield, $88 \%$ ee $\quad 3$ eh, $37 \%$ yield, $90 \%$ ee $\quad 3$ ei, $99 \%$ yield, $94 \%$ ee<smiles>O=[N+]([O-])C[C@H](c1cccs1)c1c(O)ccc2cc[nH]c12</smiles><smiles>O=[N+]([O-])C[C@H](/C=C/c1ccccc1)c1c(O)ccc2cc[nH]c12</smiles>

3ej, $92 \%$ yield, $94 \%$ ee $\quad 3$ ek, $44 \%$ yield, $89 \%$ ee

${ }^{a}$ Reaction conditions: 1e $(0.15 \mathrm{mmol}), 2(0.1 \mathrm{mmol})$, and VI $(2 \mathrm{~mol}$ $\%)$ in $1 \mathrm{~mL}$ of $\mathrm{CHCl}_{3}$ at $4{ }^{\circ} \mathrm{C}$. Isolated yields after column chromatography. Enantiomeric ratios were determined by HPLC using chiral stationary phase.

excess. 6-Hydroxyindole 1e proved to be less reactive than 1a and $\mathbf{1 b}$, and in general, we obtain lower conversions but the products were obtained with excellent enantiomeric excesses. Again, the conjugated nitrodiene derived from cinnamaldehyde 2k gave excellent regioselectivity and good ee value (89\%), although with a moderate yield (44\%).

Finally, 7-hydroxyindole 1f was also tested under the optimized reaction conditions (Scheme 5). Unfortunately, the

Scheme 5. Friedel-Crafts Alkylation of 7-Hydroxyindole if with $2 a^{a}$

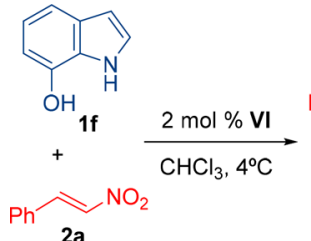

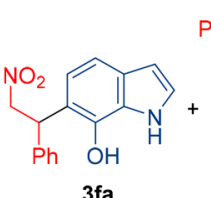

$3 \mathrm{fa}$

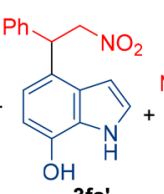

'fa'

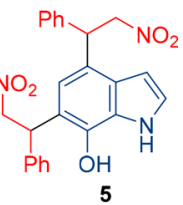

3fa:3fa':5 (53:16:32), determined by ${ }^{1} \mathrm{H}$ NMR

3fa, $45 \%$ isolated yield, $18 \%$ ee

${ }^{a}$ Reaction conditions: 1 f $(0.15 \mathrm{mmol}), \mathbf{2 a}(0.1 \mathrm{mmol})$, and VI $(2 \mathrm{~mol}$ $\%)$ in $1 \mathrm{~mL}$ of $\mathrm{CHCl}_{3}$ at $4{ }^{\circ} \mathrm{C}$. Enantiomeric excesses were determined by HPLC using chiral stationary phase.

regioselectivity was low, obtaining a ratio (53:16:32), determined by ${ }^{1} \mathrm{H}$ NMR, of product alkylated at C-6 (3da), at C-4 (3da') and also the product of double alkylation 5, and the enantiomeric excess of compound $3 \mathbf{d a}$ was also very poor ( $18 \%$ ee). We attribute these results to an interference between the $\mathrm{NH}$ of the indole nucleous and the hydroxyl group.

To rationalize the observed regio- and enantioselectivity, we propose a tentative transition state (Figure 2). The squaramide
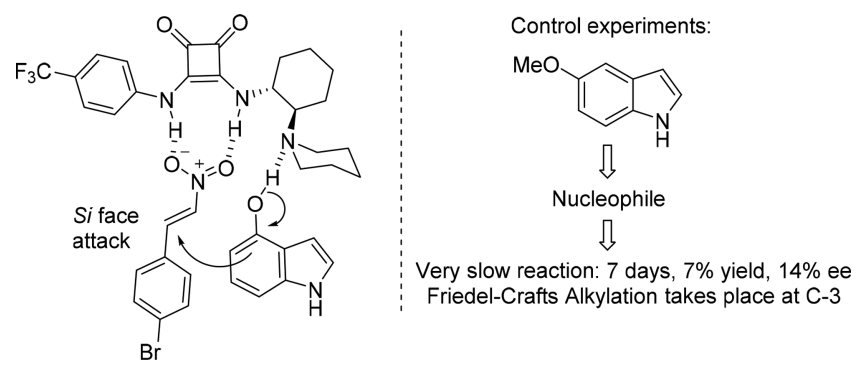

Figure 2. Proposed stereochemical model.

acts as a bifunctional organocatalyst responsible for the preorientation and the activation of the substrates. While the nitroalkene is activated upon formation of hydrogen bonds between nitro group and the squaramide, ${ }^{22}$ the hydroxyindole undergoes nucleophilic activation by hydrogen bonding with the tertiary amine moiety of the catalyst. This model explains the ortho-regioselectivity for the nucleophiles and the $S$ absolute configuration of the final products. The influence of the catalyst/OH-group interaction can be ascertained by the fact that the 5-methoxyindole reacts very slowly under the optimized reaction conditions ( $7 \%$ yield after 7 days), and the corresponding Friedel-Crafts alkylation takes place at C-3.

To showcase the utility of our catalytic protocol for the synthesis of chiral 2-amino-1,1-diarylalkanes, we performed first the reaction of 5-hydroxyindole $\mathbf{1 b}$ and nitroalkene $\mathbf{2 a}$ at $\mathbf{1}$ mmol scale, obtaining the product 3 ba with better yield (93\%) and the same ee $(96 \%)$ as when the reaction was carried out using $0.1 \mathrm{mmol}$ of $\mathbf{2 a}$ (Scheme 6A). Next, we carried out the selective reduction of the nitro group present in the alkylated indoles $3 \mathrm{ba}$ and $3 \mathrm{cc}$ with $\mathrm{NaBH}_{4}-\mathrm{NiCl}_{2} \cdot 6 \mathrm{H}_{2} \mathrm{O}$, obtaining the

\section{Scheme 6. Synthetic Transformations ${ }^{a}$}

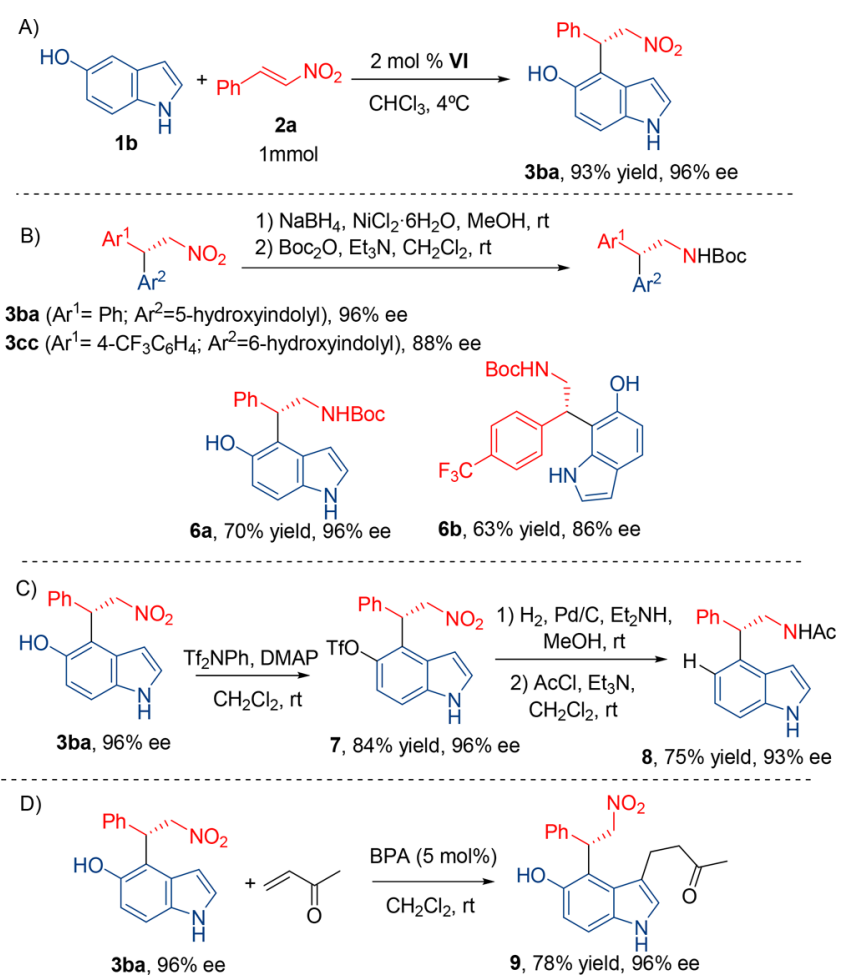

${ }^{a}$ Isolated yields after column chromatography. Enantiomeric excesses were determined by HPLC using chiral stationary phase. 
corresponding 2-amino-1,1-diarylalkanes with good yields and preserving the ee, which were isolated as Boc-derivatives $\mathbf{6 a}$ and 6b (Scheme 6B). To eliminate the activating-directing hydroxy group, we have also transformed compound $3 \mathrm{ba}$ in the corresponding triflate 7 . Triflates can be considered as privileged synthetic intermediates for the synthesis of a large number of molecules. ${ }^{23}$ So, triflate 7 was catalytically hydrogenated with $\mathrm{Pd} / \mathrm{C}$ at $1 \mathrm{~atm}$, affording the corresponding chiral 2-amino-1,1-diarylalkane 8 with a C-4 indolyl substituent in good yield and without erosion in the enantiomeric excess (Scheme 6C). In this procedure, the nitro group was simultaneously reduced to the corresponding amine, which was protected with $\mathrm{AcCl}$ to facilitate the isolation. With this transformation, we demonstrate that the activating/directing hydroxy group could be posteriorly removed obtaining the chiral hydroxy group-free indole. Finally, we performed the reaction of compound 3 ba with methylvinylketone under acidic catalysis to obtain product 9, following a classical FriedelCrafts alkylation at C-3 position of the indole scaffold, proving that our method can provide highly functionalized chiral indoles (Scheme 6D) and consequently 2-amino-1,1-diarylalkanes.

\section{CONCLUSION}

In summary, we have described an enantioselective synthesis of 2-amino-1,1-diarylalkanes bearing a carbocyclic ring substituted indole through asymmetric catalytic reaction of hydroxyindoles with nitroalkenes followed by reduction of the nitro group to amine. The asymmetric catalytic reaction described here is the first regio- and enantioselective alkylation in the carbocyclic ring of indoles with nitroalkenes as electrophiles, which was catalyzed by the commercially available Rawal squaramide VI ( 2 mol \% catalyst). Under our reaction conditions, hydroxyindoles react in the carbocyclic ring rather than in the azole system because the hydroxy group acts as an activating group but also directing group. We can introduce regioselectively the electrophile at C-4, C-5, and C-7 with high levels of enantioselectivity, by using 5-hydroxyindole, 4-hydroxyindole, and 6-hydroxyindole, respectively. ${ }^{24}$ The selective reduction of the nitro group present in the alkylated indoles was carried with $\mathrm{NaBH}_{4}-\mathrm{NiCl}_{2} \cdot 6 \mathrm{H}_{2} \mathrm{O}$, obtaining the corresponding 2-amino1,1-diarylalkanes with good yield and preserving the ee. In addition, we have performed the removal of the hydroxy group, through the corresponding triflate, simultaneously to the reduction of nitro group under mild reductive elimination conditions in high overall yield. Studies to further extend the scope of this reaction are currently underway in our laboratory.

\section{EXPERIMENTAL SECTION}

General Methods. Reactions were carried out in $5 \mathrm{~mL}$ vials under air. Comercial reagents were used as purchased. Reactions were monitored by TLC analysis using Merck Silica Gel 60 F-254 thin layer plates. Flash column chromatography was performed on Merck silica gel $60,0.040-0.063 \mathrm{~mm}$, and visualized using both a UV lamp (254 $\mathrm{nm}$ ) and then a CAM solution (an aqueous solution of ceric ammonium molybdate). Melting points were determined in capillary tubes. NMR spectra were run at $300 \mathrm{MHz}$ for ${ }^{1} \mathrm{H}$ and $75 \mathrm{MHz}$ for ${ }^{13} \mathrm{C}$ using residual nondeuterated solvent as internal standard $\left(\mathrm{CHCl}_{3}, \delta\right.$ 7.26 and $77.00 \mathrm{ppm}$, respectively; $\mathrm{MeOH}, \delta 3.34 \mathrm{ppm}$ and $\delta 49.87$ ppm, respectively). Chemical shifts are given in ppm. The carbon type was determined by DEPT experiments. High-resolution mass spectra (ESI) were recorded on a AB SCIEX Triple TOF spectrometer equipped with an electrospray source with a capillary voltage of $4.5 \mathrm{kV}$ (ESI). Specific optical rotations were measured using sodium light (D line $589 \mathrm{~nm}$ ). Chiral HPLC analyses were performed in a chromatograph equipped with a UV diode-array detector using columns with chiral stationary phases from Daicel. Catalysts I, IV, and VI were commercially available. Catalyst II was prepared from quinine using Deng's procedure. ${ }^{25}$ Catalyst III was prepared from cinchonidine using Soós' procedure. ${ }^{26}$ Catalyst $\mathbf{V}$ was prepared from quinine using Du's procedure. ${ }^{27}$ Nitroalkenes $\mathbf{2 a}-\mathbf{j}$ were commercially available. Nitroalkenes $\mathbf{2 l}$ and $\mathbf{2 k}$ were prepared following a procedure described by Lam. ${ }^{28}$ 5-Hydroxy-1-methyl-indole $1 \mathrm{c}$ was synthesized from 5-hydroxyindole $\mathbf{1 b}$ as described in the literature. ${ }^{29}$

General Procedure for the Nonenantioselective FriedelCrafts Reaction. In a $5 \mathrm{~mL}$ vial were placed the proper hydroxyindole $(1,0.1 \mathrm{mmol})$, the proper nitroalkene $(2,0.1 \mathrm{mmol})$, and $1-(3,5-$ bis(trifluoromethyl)phenyl)-3-(3-(dimethylamino)propyl) thiourea $(3.7 \mathrm{mg}, 0.01 \mathrm{mmol})$. The mixture then was dissolved in toluene (1 $\mathrm{mL}$ ), and it was stirred at room temperature for $24 \mathrm{~h}$ and purified by column chromatography using hexane:AcOEt mixtures (95:5 to 70:30) to afford the product 3 as a racemic mixture.

General Procedure for the Enantioselective Friedel-Crafts Reaction. In a $5 \mathrm{~mL}$ vial were placed the proper hydroxyindole (1, $0.15 \mathrm{mmol})$, the proper nitroalkene $(2,0.10 \mathrm{mmol})$, and Rawal squaramide (VI, $0.84 \mathrm{mg}, 0.002 \mathrm{mmol}$ ). The mixture then was dissolved in $\mathrm{CHCl}_{3}(1 \mathrm{~mL})$, and it was stirred at $4{ }^{\circ} \mathrm{C}$ until the reaction was complete (TLC). Finally, the reaction mixture was directly poured into the column chromatography, using hexane:AcOEt mixtures (95:5 to $70: 3$ ) as eluent to afford the product 3 as an enantioenriched mixture.

Specific Procedure for the Enantioselective Friedel-Crafts Reaction with Nitroalkene 2l. In a $5 \mathrm{~mL}$ flask equipped with a refrigerant were placed 5-hydroxyindole $(\mathbf{1 b}, 0.15 \mathrm{mmol}),(E)-(4-$ nitrobut-3-en-1-yl)benzene (2l, $0.10 \mathrm{mmol})$, and Rawal Squaramide (VI, $2.1 \mathrm{mg}, 0.005 \mathrm{mmol}$ ). The mixture then was dissolved in $\mathrm{CHCl}_{3}$ $(1 \mathrm{~mL})$, and it was stirred at $50{ }^{\circ} \mathrm{C}$ until the reaction was complete (TLC). Finally, the reaction mixture was directly poured into the column chromatography, using hexane:AcOEt mixtures (95:5 to 70:3) as eluent to afford the product $3 \mathrm{al}$ as an enantioenriched mixture.

Scope of the Reaction with 4-Hydroxyindole. (S)-5-(1-Phenyl2-nitroethyl)-1H-indol-4-ol (3aa). The product 3aa was obtained as a dark oil (22.6 mg, $0.08 \mathrm{mmol}, 80 \%$ yield). Enantiomeric excess (92\%) was determined by chiral HPLC (Chiralpak AD-H), hexane- ${ }^{i} \mathrm{PrOH}$ $70: 30,1.0 \mathrm{~mL} / \mathrm{min}$, major enantiomer $t_{\mathrm{r}}=14.55 \mathrm{~min}$, minor enantiomer $t_{\mathrm{r}}=16.10 \mathrm{~min} .[\alpha]_{\mathrm{D}}^{20}-79.2\left(c=0.55, \mathrm{CHCl}_{3}\right)(92 \%$ ee); ${ }^{1} \mathrm{H}$ NMR $\left(300 \mathrm{MHz}, \mathrm{CDCl}_{3}\right) \delta 8.16(\mathrm{~s}, 1 \mathrm{H}), 7.38-7.23(\mathrm{~m}, 4 \mathrm{H})$, $7.26-7.15(\mathrm{~m}, 1 \mathrm{H}), 7.09(\mathrm{dd}, J=3.3,2.3 \mathrm{~Hz}, 1 \mathrm{H}), 6.93(\mathrm{dd}, J=8.4$, $0.7 \mathrm{~Hz}, 1 \mathrm{H}), 6.89$ (d, $J=8.5 \mathrm{~Hz}, 1 \mathrm{H}), 6.49-6.40(\mathrm{~m}, 1 \mathrm{H}), 5.36(\mathrm{dd}, J$ $=8.7,7.5 \mathrm{~Hz}, 1 \mathrm{H}), 5.18(\mathrm{~s}, 1 \mathrm{H}), 5.17-5.03(\mathrm{~m}, 2 \mathrm{H}) ;{ }^{13} \mathrm{C}$ NMR $(75$ $\left.\mathrm{MHz}, \mathrm{CDCl}_{3}\right) 145.9$ (C), 139.8 (C), 136.7 (C), $128.7(\mathrm{CH}), 127.8$ $(\mathrm{CH}), 127.1(\mathrm{CH}), 123.8(\mathrm{CH}), 122.7(\mathrm{CH}), 118.0(\mathrm{C}), 114.8(\mathrm{C})$, $104.6(\mathrm{CH}), 97.8(\mathrm{CH}), 78.5\left(\mathrm{CH}_{2}\right), 43.2(\mathrm{CH})$; HRMS (ESI) $\mathrm{m} / z$ : $[\mathrm{M}+\mathrm{H}]^{+}$calcd for $\mathrm{C}_{16} \mathrm{H}_{15} \mathrm{~N}_{2} \mathrm{O}_{3}, 283.1077$; found, 283.1083 .

(S)-5-(1-(4-Bromophenyl)-2-nitroethyl)-1H-indol-4-ol (3ab). The product $3 \mathbf{a b}$ was obtained as a yellow solid $(35.0 \mathrm{mg}, 0.097 \mathrm{mmol}$, $97 \%$ yield); mp $133-138{ }^{\circ} \mathrm{C}$. Enantiomeric excess ( $87 \%$ and $90 \%$ after crystallization) was determined by chiral HPLC (Chiralpak AD-H), hexane $-{ }^{i} \operatorname{PrOH} 80: 20,1.0 \mathrm{~mL} / \mathrm{min}$, major enantiomer $t_{\mathrm{r}}=28.39 \mathrm{~min}$, minor enantiomer $t_{\mathrm{r}}=31.20 \mathrm{~min}$. $[\alpha]_{\mathrm{D}}^{20}-45.2\left(c=0.29, \mathrm{CHCl}_{3}\right)(87 \%$ ee); ${ }^{1} \mathrm{H}$ NMR $\left(300 \mathrm{MHz}, \mathrm{CDCl}_{3}\right) \delta 8.19(\mathrm{~s}, 1 \mathrm{H}), 7.41(\mathrm{~d}, J=8.5 \mathrm{~Hz}$, $2 \mathrm{H}), 7.20(\mathrm{~d}, J=8.3 \mathrm{~Hz}, 2 \mathrm{H}), 7.14(\mathrm{dd}, J=3.3,2.3 \mathrm{~Hz}, 1 \mathrm{H}), 6.95(\mathrm{dd}$, $J=8.4,0.9 \mathrm{~Hz}, 1 \mathrm{H}), 6.85(\mathrm{~d}, J=8.4 \mathrm{~Hz}, 1 \mathrm{H}), 6.46(\mathrm{ddd}, J=3.2,2.0$, $0.9 \mathrm{~Hz}, 1 \mathrm{H}), 5.30(\mathrm{dd}, J=9.0,7.2 \mathrm{~Hz}, 1 \mathrm{H}), 5.13(\mathrm{~s}, 1 \mathrm{H}), 5.11-5.03$ $(\mathrm{m}, 2 \mathrm{H}) ;{ }^{13} \mathrm{C}$ NMR $\left(75 \mathrm{MHz}, \mathrm{CDCl}_{3}\right) \delta 146.7(\mathrm{C}), 138.8(\mathrm{C}), 131.7$ $(\mathrm{CH}), 131.2(\mathrm{C}), 129.4(\mathrm{CH}), 128.2(\mathrm{C}), 125.6(\mathrm{CH}), 120.9(\mathrm{C})$, $115.0(\mathrm{C}), 112.9(\mathrm{CH}), 111.3(\mathrm{CH}), 100.6(\mathrm{CH}), 77.7\left(\mathrm{CH}_{2}\right), 42.9$ $(\mathrm{CH})$; HRMS (ESI) $m / z:[\mathrm{M}+\mathrm{H}]^{+}$calcd for $\mathrm{C}_{16} \mathrm{H}_{14} \mathrm{~N}_{2} \mathrm{O}_{3} \mathrm{Br}$, 361.0182; found, 361.0192 .

(S)-5-(1-(4-(Trifluoromethyl)phenyl)-2-nitroethyl)-1 $\mathrm{H}$-indol-4-ol (3ac). The product $3 \mathrm{ac}$ was obtained as a dark oil $(30.1 \mathrm{mg}, 0.086$ mmol, $86 \%$ yield). Enantiomeric excess (89\%) was determined by chiral HPLC (Chiralpak AD-H), hexane- ${ }^{i} \mathrm{PrOH} 80: 20,1.0 \mathrm{~mL} / \mathrm{min}$, major enantiomer $t_{\mathrm{r}}=19.35 \mathrm{~min}$, minor enantiomer $t_{\mathrm{r}}=17.81 \mathrm{~min}$. 
$[\alpha]_{\mathrm{D}}^{20}-55.2\left(c=0.65, \mathrm{CHCl}_{3}\right)(89 \%$ ee $) ;{ }^{1} \mathrm{H}$ NMR $(300 \mathrm{MHz}$, $\left.\mathrm{CDCl}_{3}\right) \delta 8.20(\mathrm{~s}, 1 \mathrm{H}), 7.54(\mathrm{~d}, J=8.0 \mathrm{~Hz}, 2 \mathrm{H}), 7.45(\mathrm{~d}, J=8.2 \mathrm{~Hz}$, $2 \mathrm{H}), 7.15(\mathrm{t}, J=2.8 \mathrm{~Hz}, 1 \mathrm{H}), 6.96(\mathrm{dd}, J=8.4,0.8 \mathrm{~Hz}, 1 \mathrm{H}), 6.86(\mathrm{~d}, J$ $=8.4 \mathrm{~Hz}, 1 \mathrm{H}), 6.46(\mathrm{~s}, 1 \mathrm{H}), 5.40(\mathrm{t}, J=8.1 \mathrm{~Hz}, 1 \mathrm{H}), 5.16(\mathrm{~s}, 1 \mathrm{H}), 5.13$ $(\mathrm{d}, J=8.0 \mathrm{~Hz}, 2 \mathrm{H}) ;{ }^{13} \mathrm{C}$ NMR $\left(75 \mathrm{MHz}, \mathrm{CDCl}_{3}\right) 145.9$ (C), 144.1 (C), $136.8(\mathrm{C}), 129.3\left(\mathrm{q}, J_{\mathrm{C}-\mathrm{F}}=32.4 \mathrm{~Hz}, \mathrm{C}\right), 128.2(\mathrm{CH}), 125.6(\mathrm{q}$, $\left.J_{\mathrm{C}-\mathrm{F}}=3.8 \mathrm{~Hz}, \mathrm{CH}\right), 124.1(\mathrm{CH}), 124.4\left(\mathrm{~d}, J_{\mathrm{C}-\mathrm{F}}=272.1 \mathrm{~Hz}, \mathrm{CF}_{3}\right)$, $122.7(\mathrm{CH}), 117.9(\mathrm{C}), 114.0(\mathrm{C}), 104.8(\mathrm{CH}), 97.7(\mathrm{CH}), 78.0$ $\left(\mathrm{CH}_{2}\right), 43.3(\mathrm{CH}) ;{ }^{19} \mathrm{~F} \mathrm{NMR}\left(282.4 \mathrm{MHz}, \mathrm{CDCl}_{3}\right) \delta-63.0\left(\mathrm{~s}, \mathrm{CF}_{3}\right)$; HRMS (ESI) $m / z$ : $[\mathrm{M}+\mathrm{H}]^{+}$calcd for $\mathrm{C}_{17} \mathrm{H}_{14} \mathrm{~N}_{2} \mathrm{O}_{3} \mathrm{~F}_{3}, 351.0951$; found, 351.0963 .

(S)-5-(1-(4-Methoxyphenyl)-2-nitroethyl)-1H-indol-4-ol (3ad). The product 3ad was obtained as a green solid (24.1 mg, 0.077 mmol, 77\% yield); mp 169-173. Enantiomeric excess (86\%) was determined by chiral HPLC (Chiralpak AS-H), hexane $-{ }^{i} \mathrm{PrOH}$ 80:20, $1.0 \mathrm{~mL} / \mathrm{min}$, major enantiomer $t_{\mathrm{r}}=28.81 \mathrm{~min}$, minor enantiomer $t_{\mathrm{r}}=$ 41.49 min. $[\alpha]_{\mathrm{D}}^{20}-48.9(c=1.16, \mathrm{MeOH})(86 \% \mathrm{ee}) ;{ }^{1} \mathrm{H}$ NMR $(300$ $\left.\mathrm{MHz}, \mathrm{CDCl}_{3}\right) \delta 8.16(\mathrm{~s}, 1 \mathrm{H}), 7.24(\mathrm{~d}, J=8.3 \mathrm{~Hz}, 2 \mathrm{H}), 7.13(\mathrm{dd}, J=$ 3.4, $2.4 \mathrm{~Hz}, 1 \mathrm{H}), 6.95(\mathrm{dd}, J=8.5,0.9 \mathrm{~Hz}, 1 \mathrm{H}), 6.89(\mathrm{~d}, J=8.4 \mathrm{~Hz}$, $1 \mathrm{H}), 6.83(\mathrm{~d}, J=8.8 \mathrm{~Hz}, 2 \mathrm{H}), 6.47(\mathrm{td}, J=2.3,1.1 \mathrm{~Hz}, 1 \mathrm{H}), 5.28(\mathrm{dd}$, $J=8.7,7.5 \mathrm{~Hz}, 1 \mathrm{H}), 5.14-5.06(\mathrm{~m}, 2 \mathrm{H}), 5.02(\mathrm{dd}, J=12.7,8.9 \mathrm{~Hz}$, 1H), $3.75(\mathrm{~s}, 3 \mathrm{H}) ;{ }^{13} \mathrm{C}$ NMR (75 MHz, $\left.\mathrm{CDCl}_{3}\right) \delta 158.7$ (C), 145.9 (C), $136.7(\mathrm{C}), 131.7(\mathrm{C}), 128.8(\mathrm{CH}), 123.8(\mathrm{CH}), 122.7(\mathrm{CH})$, 118.1 (C), 115.1 (C), $114.2(\mathrm{CH}), 104.5(\mathrm{CH}), 97.9(\mathrm{CH}), 78.8$ $\left(\mathrm{CH}_{2}\right), 55.2\left(\mathrm{CH}_{3}\right), 42.6(\mathrm{CH})$; HRMS (ESI) $\mathrm{m} / z$ : $[\mathrm{M}+\mathrm{H}]^{+}$calcd for $\mathrm{C}_{17} \mathrm{H}_{17} \mathrm{~N}_{2} \mathrm{O}_{4}, 313.1183$; found, 313.1192 .

(S)-5-(1-(4-(Trifluoromethoxy)phenyl)-2-nitroethyl)-1H-indol-4-ol (3ae). The product 3ae was obtained as a dark oil (34.8 mg, 0.095 mmol, 95\% yield). Enantiomeric excess (88\%) was determined by chiral HPLC (Chiralpak AD-H), hexane- ${ }^{i}$ PrOH 80:20, $1.0 \mathrm{~mL} / \mathrm{min}$, major enantiomer $t_{\mathrm{r}}=17.28 \mathrm{~min}$, minor enantiomer $t_{\mathrm{r}}=14.72 \mathrm{~min}$. $[\alpha]_{\mathrm{D}}^{20}-39.2\left(c=0.91, \mathrm{CHCl}_{3}\right)(88 \%$ ee $) ;{ }^{1} \mathrm{H}$ NMR $(300 \mathrm{MHz}$, $\left.\mathrm{CDCl}_{3}\right) \delta 8.20(\mathrm{~s}, 1 \mathrm{H}), 7.36(\mathrm{~d}, J=8.6 \mathrm{~Hz}, 2 \mathrm{H}), 7.15-7.06(\mathrm{~m}, 3 \mathrm{H})$, $6.96(\mathrm{~d}, J=8.5 \mathrm{~Hz}, 1 \mathrm{H}), 6.88(\mathrm{~d}, J=8.4 \mathrm{~Hz}, 1 \mathrm{H}), 6.57-6.40(\mathrm{~m}, 1 \mathrm{H})$, $5.35(\mathrm{dd}, J=8.9,7.3 \mathrm{~Hz}, 1 \mathrm{H}), 5.20(\mathrm{~s}, 1 \mathrm{H}), 5.16-5.01(\mathrm{~m}, 2 \mathrm{H}) ;{ }^{13} \mathrm{C}$ NMR (75 MHz, $\mathrm{CDCl}_{3}$ ) $\delta 148.2$ (C), 145.9 (C), 138.7 (C), 136.8 (C), $129.2(\mathrm{CH}), 124.0(\mathrm{CH}), 122.7(\mathrm{CH}), 121.1(\mathrm{CH}), 120.4(\mathrm{~d}$, $\left.J_{\mathrm{C}-\mathrm{F}}=257.1 \mathrm{~Hz}, \mathrm{CF}_{3}\right), 118.0(\mathrm{C}), 114.3(\mathrm{C}), 104.7(\mathrm{CH}), 97.8(\mathrm{CH})$, $78.3\left(\mathrm{CH}_{2}\right), 42.9(\mathrm{CH}) ;{ }^{19} \mathrm{~F}$ NMR $\left(282.4 \mathrm{MHz}, \mathrm{CDCl}_{3}\right) \delta-58.3(\mathrm{~s}$, $\mathrm{CF}_{3}$ ); HRMS (ESI) $m / z:[\mathrm{M}+\mathrm{H}]^{+}$calcd for $\mathrm{C}_{17} \mathrm{H}_{14} \mathrm{~N}_{2} \mathrm{O}_{4} \mathrm{~F}_{3}$, 367.0900; found, 367.0916.

(S)-5-(1-(o-Tolyl)-2-nitroethyl)-1H-indol-4-ol (3af). The product 3af was obtained as a green solid $(17.2 \mathrm{mg}, 0.058 \mathrm{mmol}, 58 \%$ yield); mp 160-165. Enantiomeric excess (98\%) was determined by chiral HPLC (Chiralpak IC), hexane- ${ }^{i} \mathrm{PrOH} 80: 20,1.0 \mathrm{~mL} / \mathrm{min}$, major enantiomer $t_{\mathrm{r}}=4.88 \mathrm{~min}$, minor enantiomer $t_{\mathrm{r}}=7.33 \mathrm{~min} .[\alpha]_{\mathrm{D}}^{20}-65.9$ $\left(c=0.44, \mathrm{CHCl}_{3}\right)\left(98 \%\right.$ ee); ${ }^{1} \mathrm{H} \mathrm{NMR}\left(300 \mathrm{MHz}, \mathrm{CDCl}_{3}\right) \delta 8.15(\mathrm{~s}$, $1 \mathrm{H}), 7.34(\mathrm{~d}, J=7.0 \mathrm{~Hz}, 1 \mathrm{H}), 7.23-7.11(\mathrm{~m}, 4 \mathrm{H}), 6.89(\mathrm{dd}, J=8.5$, $0.7 \mathrm{~Hz}, 1 \mathrm{H}), 6.75(\mathrm{~d}, J=8.5 \mathrm{~Hz}, 1 \mathrm{H}), 6.48(\mathrm{ddd}, J=3.0,1.9,0.8 \mathrm{~Hz}$, $1 \mathrm{H}), 5.59-5.52(\mathrm{~m}, 1 \mathrm{H}), 5.23(\mathrm{~s}, 1 \mathrm{H}), 5.03-4.99(\mathrm{~m}, 2 \mathrm{H}), 2.28(\mathrm{~s}$, $3 \mathrm{H}) ;{ }^{13} \mathrm{C}$ NMR (75 MHz, $\left.\mathrm{CDCl}_{3}\right) \delta 146.0(\mathrm{C}), 137.6(\mathrm{C}), 137.1(\mathrm{C})$, $136.7(\mathrm{C}), 131.1(\mathrm{CH}), 127.1(\mathrm{CH}), 126.1(\mathrm{CH}), 125.9(\mathrm{CH}), 123.8$ $(\mathrm{CH}), 122.9(\mathrm{CH}), 117.8(\mathrm{C}), 113.9(\mathrm{C}), 104.6(\mathrm{CH}), 97.9(\mathrm{CH})$, $77.9\left(\mathrm{CH}_{2}\right), 38.9(\mathrm{CH}), 19.4\left(\mathrm{CH}_{3}\right)$; HRMS (ESI) $m / z:[\mathrm{M}+\mathrm{H}]^{+}$ calcd for $\mathrm{C}_{17} \mathrm{H}_{17} \mathrm{~N}_{2} \mathrm{O}_{3}, 297.1234$; found, 297.1225.

(S)-5-(1-(2,4-Dimethoxyphenyl)-2-nitroethyl)-1H-indol-4-ol (3ag). The product $3 \mathrm{ag}$ was obtained as a dark oil $(27.7 \mathrm{mg}, 0.081 \mathrm{mmol}$, $81 \%$ yield). Enantiomeric excess $(79 \%)$ was determined by chiral HPLC (Chiralpak AD-H), hexane- $-^{i} \mathrm{PrOH}$ 80:20, $1.0 \mathrm{~mL} / \mathrm{min}$, major enantiomer $t_{\mathrm{r}}=48.84 \mathrm{~min}$, minor enantiomer $t_{\mathrm{r}}=30.42 \mathrm{~min}$. $[\alpha]_{\mathrm{D}}^{20}$ -73.2 ( $\left.c=0.55, \mathrm{CHCl}_{3}\right)\left(79 \%\right.$ ee); ${ }^{1} \mathrm{H}$ NMR $\left(300 \mathrm{MHz}, \mathrm{CDCl}_{3}\right) \delta$ $8.12(\mathrm{~s}, 1 \mathrm{H}), 7.12(\mathrm{~d}, J=8.3 \mathrm{~Hz}, 1 \mathrm{H}), 7.07(\mathrm{dd}, J=3.3,2.3 \mathrm{~Hz}, 1 \mathrm{H})$, 6.98-6.89 (m, 2H), 6.53 (ddd, $J=3.1,2.1,0.8 \mathrm{~Hz}, 1 \mathrm{H}), 6.47-6.39$ $(\mathrm{m}, 2 \mathrm{H}), 6.10(\mathrm{~s}, 1 \mathrm{H}), 5.51(\mathrm{t}, J=8.2 \mathrm{~Hz}, 1 \mathrm{H}), 5.19-4.95(\mathrm{~m}, 2 \mathrm{H})$, $3.87(\mathrm{~s}, 3 \mathrm{H}), 3.74(\mathrm{~s}, 3 \mathrm{H}) ;{ }^{13} \mathrm{C} \mathrm{NMR}\left(75 \mathrm{MHz}, \mathrm{CDCl}_{3}\right) \delta 160.0(\mathrm{C})$, $157.1(\mathrm{C}), 146.5(\mathrm{C}), 136.7(\mathrm{C}), 128.3(\mathrm{CH}), 123.5(\mathrm{CH}), 121.5$ (CH), $120.1(\mathrm{C}), 118.5(\mathrm{C}), 114.3(\mathrm{C}), 105.1(\mathrm{CH}), 104.4(\mathrm{CH})$, $99.1(\mathrm{CH}), 99.0(\mathrm{CH}), 77.8\left(\mathrm{CH}_{2}\right), 55.9\left(\mathrm{CH}_{3}\right), 55.4\left(\mathrm{CH}_{3}\right), 35.8$ (CH); HRMS (ESI) $m / z$ : $[\mathrm{M}+\mathrm{H}]^{+}$calcd for $\mathrm{C}_{18} \mathrm{H}_{19} \mathrm{~N}_{2} \mathrm{O}_{5}, 343.1288$; found, 343.1285 .
(S)-5-(1-(4-Hydroxy-3-methoxyphenyl)-2-nitroethyl)-1H-indol-4ol (3ah). The product 3ah was obtained as a dark oil $(27.2 \mathrm{mg}, 0.075$ mmol, $75 \%$ yield). Enantiomeric excess (94\%) was determined by chiral HPLC (Chiralpak AD-H), hexane- ${ }^{i} \mathrm{PrOH} 70: 30,1.0 \mathrm{~mL} / \mathrm{min}$, major enantiomer $t_{\mathrm{r}}=27.50 \mathrm{~min}$, minor enantiomer $t_{\mathrm{r}}=17.22 \mathrm{~min}$. $[\alpha]_{\mathrm{D}}^{20}-78.2\left(c=0.47, \mathrm{CHCl}_{3}\right)(94 \%$ ee $) ;{ }^{1} \mathrm{H}$ NMR $(300 \mathrm{MHz}$, $\left.\mathrm{CDCl}_{3}\right) \delta 8.18(\mathrm{bs}, 1 \mathrm{H}), 7.12(\mathrm{dd}, J=3.3,2.4 \mathrm{~Hz}, 1 \mathrm{H}), 6.94(\mathrm{dd}, J=$ $8.5,0.9 \mathrm{~Hz}, 1 \mathrm{H}), 6.88(\mathrm{~s}, J=8.4 \mathrm{~Hz}, 1 \mathrm{H}), 6.84(\mathrm{~m}, 2 \mathrm{H}), 6.80(\mathrm{~s}, 1 \mathrm{H})$, $6.48(\mathrm{ddd}, J=3.2,2.1,0.9 \mathrm{~Hz}, 1 \mathrm{H}), 5.53(\mathrm{bs}, 1 \mathrm{H}), 5.30-5.22(\mathrm{~m}, 2 \mathrm{H})$, 5.12-4.97 (m, 2H), $3.80(\mathrm{~s}, 3 \mathrm{H}) ;{ }^{13} \mathrm{C}$ NMR $\left(75 \mathrm{MHz} \mathrm{CDCl}_{3}\right) \delta$ 146.7 (C), 145.9 (C), 144.8 (C), 136.7 (C), $131.6(\mathrm{C}), 123.9(\mathrm{CH})$, $122.6(\mathrm{CH}), 120.1(\mathrm{CH}), 118.1(\mathrm{C}), 115.0(\mathrm{C}), 114.5(\mathrm{CH}), 110.8$ $(\mathrm{CH}), 104.5(\mathrm{CH}), 98.0(\mathrm{CH}), 78.7\left(\mathrm{CH}_{2}\right), 55.9\left(\mathrm{CH}_{3}\right), 43.0(\mathrm{CH})$; HRMS (ESI) $m / z:[\mathrm{M}+\mathrm{H}]^{+}$calcd for $\mathrm{C}_{17} \mathrm{H}_{17} \mathrm{~N}_{2} \mathrm{O}_{5}, 329.1132$; found, 329.1143.

(S)-5-(1-(Furan-2-yl)-2-nitroethyl)-1H-indol-4-ol (3ai). The product 3ai was obtained as a yellow oil $(24.5 \mathrm{mg}, 0.09 \mathrm{mmol}, 90 \%$ yield). Enantiomeric excess (95\%) was determined by chiral HPLC (Chiralpak AD-H), hexane- ${ }^{i} \mathrm{PrOH} 80: 20,1.0 \mathrm{~mL} / \mathrm{min}$, major enantiomer $t_{\mathrm{r}}=23.48 \mathrm{~min}$, minor enantiomer $t_{\mathrm{r}}=25.76 \mathrm{~min}$. $[\alpha]_{\mathrm{D}}^{20}$ $-52.14\left(c=0.66, \mathrm{CHCl}_{3}\right)(95 \% \mathrm{ee}) ;{ }^{1} \mathrm{H} \mathrm{NMR}\left(300 \mathrm{MHz}, \mathrm{CDCl}_{3}\right) \delta$ $7.191-7.151(\mathrm{~m}, 3 \mathrm{H}), 7.02(\mathrm{dt}, J=3.5,1.1 \mathrm{~Hz}, 1 \mathrm{H}), 6.91(\mathrm{dd}, J=5.1$, $3.6 \mathrm{~Hz}, 1 \mathrm{H}), 6.67(\mathrm{~d}, J=8.6 \mathrm{~Hz}, 1 \mathrm{H}), 6.52$ (ddd, $J=3.1,2.1,0.9 \mathrm{~Hz}$, $1 \mathrm{H}), 5.72(\mathrm{td}, J=7.7,0.9 \mathrm{~Hz}, 1 \mathrm{H}), 5.38(\mathrm{dd}, J=13.0,7.8 \mathrm{~Hz}, 1 \mathrm{H})$, $5.27(\mathrm{dd}, J=13.0,7.5 \mathrm{~Hz}, 1 \mathrm{H}), 4.82(\mathrm{bs}, 1 \mathrm{H}) ;{ }^{13} \mathrm{C}$ NMR $(75.5 \mathrm{MHz}$, $\left.\mathrm{CDCl}_{3}\right) \delta 152.8(\mathrm{C}), 146.1(\mathrm{C}), 142.1(\mathrm{CH}), 137.0(\mathrm{C}), 123.9(\mathrm{CH})$, $123.0(\mathrm{CH}), 118.0(\mathrm{C}), 112.4(\mathrm{C}), 110.4(\mathrm{CH}), 107.1(\mathrm{CH}), 104.7$ (CH), 98.1 (CH), $77.2\left(\mathrm{CH}_{2}\right), 37.7(\mathrm{CH})$; HRMS (ESI) $m / z:[\mathrm{M}+$ $\mathrm{H}]^{+}$calcd for $\mathrm{C}_{14} \mathrm{H}_{13} \mathrm{~N}_{2} \mathrm{O}_{4}, 273.0870$; found, 273.0877.

(S)-5-(2-Nitro-1-(thiophen-2-yl)ethyl)-1H-indol-4-ol (3aj). The product 3aj was obtained as a colorless oil $(26.8 \mathrm{mg}, 0.093 \mathrm{mmol}$, $93 \%$ yield). Enantiomeric excess (91\%) was determined by chiral HPLC (Chiralpak AD-H), hexane- ${ }^{i} \mathrm{PrOH}$ 80:20, $1.0 \mathrm{~mL} / \mathrm{min}$, major enantiomer $t_{\mathrm{r}}=20.50 \mathrm{~min}$, minor enantiomer $t_{\mathrm{r}}=34.27 \mathrm{~min}$. $[\alpha]_{\mathrm{D}}^{20}$ $-0.15\left(c=1.45, \mathrm{CHCl}_{3}\right)(91 \%$ ee $) ;{ }^{1} \mathrm{H}$ NMR $\left(300 \mathrm{MHz}, \mathrm{CDCl}_{3}\right) \delta$ 8.21 (bs, $1 \mathrm{H}), 7.19(\mathrm{dd}, J=5.1,1.3 \mathrm{~Hz}, 1 \mathrm{H}), 7.12(\mathrm{dd}, J=3.3,2.4 \mathrm{~Hz}$, $1 \mathrm{H}), 7.04-6.89(\mathrm{~m}, 4 \mathrm{H}), 6.48(\mathrm{dd}, J=2.8,2.0 \mathrm{~Hz}, 1 \mathrm{H}), 5.69-5.51$ $(\mathrm{m}, 1 \mathrm{H}), 5.32(\mathrm{bs}, 1 \mathrm{H}), 5.12-5.04(\mathrm{~m}, 2 \mathrm{H}) ;{ }^{13} \mathrm{C}$ NMR $(75.5 \mathrm{MHz}$, $\left.\mathrm{CDCl}_{3}\right) \delta 145.9(\mathrm{C}), 143.4(\mathrm{C}), 136.9(\mathrm{C}), 126.8(\mathrm{CH}), 124.9(\mathrm{CH})$, $124.8(\mathrm{CH}), 124.0(\mathrm{CH}), 122.7(\mathrm{CH}), 118.0(\mathrm{C}), 114.4(\mathrm{C}), 104.3$ $(\mathrm{CH}), 98.0(\mathrm{CH}), 79.2\left(\mathrm{CH}_{2}\right), 39.1(\mathrm{CH})$; HRMS (ESI) $\mathrm{m} / \mathrm{z}$ : $[\mathrm{M}+$ $\mathrm{H}]^{+}$calcd for $\mathrm{C}_{14} \mathrm{H}_{13} \mathrm{~N}_{2} \mathrm{O}_{3} \mathrm{~S}$, 289.0641; found, 289.0650 .

(S,E)-5-(1-Nitro-4-phenylbut-3-en-2-yl)-1H-indol-4-ol (3ak). The product 3ak was obtained as a colorless oil $(26.3 \mathrm{mg}, 0.085 \mathrm{mmol}$, $85 \%$ yield). Enantiomeric excess (89\%) was determined by chiral HPLC (Chiralpak AS-H), hexane $-{ }^{i}$ PrOH 80:20, $1.0 \mathrm{~mL} / \mathrm{min}$, major enantiomer $t_{\mathrm{r}}=19.36 \mathrm{~min}$, minor enantiomer $t_{\mathrm{r}}=21.63 \mathrm{~min}$. $[\alpha]_{\mathrm{D}}^{20}$ $-17.79\left(c=1.04, \mathrm{CHCl}_{3}\right)(89 \%$ ee $) ;{ }^{1} \mathrm{H}$ NMR $\left(300 \mathrm{MHz}, \mathrm{CDCl}_{3}\right) \delta$ 8.21 (bs, $1 \mathrm{H}), 7.52-7.19(\mathrm{~m}, 5 \mathrm{H}), 7.14(\mathrm{dd}, J=3.3,2.4 \mathrm{~Hz}, 1 \mathrm{H})$, 7.06-6.94 (m, 2H), 6.57-6.54 (m, 2H), 6.53-6.50 (m, 1H), 5.38 (bs, $1 \mathrm{H}), 4.94-4.87(\mathrm{~m}, 2 \mathrm{H}), 4.77$ (ddd, $J=8.7,4.3,2.1 \mathrm{~Hz}, 1 \mathrm{H}) ;{ }^{13} \mathrm{C}$ NMR (75.5 MHz, $\left.\mathrm{CDCl}_{3}\right) \delta 146.1$ (C), 136.8 (C), 136.7 (C), 132.2 $(\mathrm{CH}), 128.5(\mathrm{CH}), 127.6(\mathrm{CH}), 127.3(\mathrm{CH}), 126.4(\mathrm{CH}), 123.9$ $(\mathrm{CH}), 123.1(\mathrm{CH}), 118.1(\mathrm{C}), 113.6(\mathrm{C}), 104.7(\mathrm{CH}), 97.8(\mathrm{CH})$, $78.9\left(\mathrm{CH}_{2}\right), 42.8(\mathrm{CH})$; HRMS (ESI) $m / z:[\mathrm{M}+\mathrm{H}]^{+}$calcd for $\mathrm{C}_{18} \mathrm{H}_{17} \mathrm{~N}_{2} \mathrm{O}_{3}, 309.1234$; found, 309.1237.

Scope of the Reaction with 5-Hydroxyindole. (S)-4-(1-Phenyl2-nitroethyl)- $1 \mathrm{H}$-indol-5-ol (3ba). The product 3 ba was obtained as a dark oil ( $0.1 \mathrm{mmol}$ scale reaction, $22.4 \mathrm{mg}$, $0.079 \mathrm{mmol}$, 79\% yield; 1 mmol scale reaction, $262.7 \mathrm{mg}, 0.93 \mathrm{mmol}$, $93 \%$ yield). Enantiomeric excess (96\%) was determined by chiral HPLC (Chiralpak AD-H), hexane $-{ }^{i} \operatorname{PrOH} 80: 20,1.0 \mathrm{~mL} / \mathrm{min}$, major enantiomer $t_{\mathrm{r}}=15.30 \mathrm{~min}$, minor enantiomer $t_{\mathrm{r}}=13.88 \mathrm{~min}$. $[\alpha]_{\mathrm{D}}^{20}-48.8\left(c=1.07, \mathrm{CHCl}_{3}\right)(96 \%$ ee); ${ }^{1} \mathrm{H}$ NMR (300 MHz, $\left.\mathrm{CDCl}_{3}\right) \delta 8.07$ (bs, $\left.1 \mathrm{H}\right), 7.43-7.36(\mathrm{~m}$, $2 \mathrm{H}), 7.30-7.25(\mathrm{~m}, 2 \mathrm{H}), 7.27-7.10(\mathrm{~m}, 3 \mathrm{H}), 6.63(\mathrm{~d}, J=8.7 \mathrm{~Hz}$, $1 \mathrm{H}), 6.51(\mathrm{ddd}, J=3.2,2.1,1.0 \mathrm{~Hz}, 1 \mathrm{H}), 5.51-5.21(\mathrm{~m}, 3 \mathrm{H}), 4.68(\mathrm{bs}$, $1 \mathrm{H}) ;{ }^{13} \mathrm{C}$ NMR (75 MHz, $\left.\mathrm{CDCl}_{3}\right) \delta 146.9(\mathrm{C}), 139.6(\mathrm{C}), 131.3(\mathrm{C})$, $128.7(\mathrm{CH}), 128.4(\mathrm{C}), 127.6(\mathrm{CH}), 127.1(\mathrm{CH}), 125.4(\mathrm{CH}), 115.7$ (C), $113.1(\mathrm{CH}), 111.1(\mathrm{CH}), 100.8(\mathrm{CH}), 77.9\left(\mathrm{CH}_{2}\right), 43.3(\mathrm{CH})$; 
HRMS (ESI) $m / z:[\mathrm{M}+\mathrm{H}]^{+}$calcd for $\mathrm{C}_{16} \mathrm{H}_{15} \mathrm{~N}_{2} \mathrm{O}_{3}, 283.1077$; found, 283.1089 .

(S)-4-(1-(4-Bromophenyl)-2-nitroethyl)-1H-indol-5-ol (3bb). The product $3 \mathbf{b b}$ was obtained as a dark oil $(34.3 \mathrm{mg}, 0.095 \mathrm{mmol}, 95 \%$ yield). Enantiomeric excess (96\%) was determined by chiral HPLC (Chiralpak AD-H), hexane- ${ }^{i} \mathrm{PrOH} 80: 20,1.0 \mathrm{~mL} / \mathrm{min}$, major enantiomer $t_{\mathrm{r}}=13.90 \mathrm{~min}$, minor enantiomer $t_{\mathrm{r}}=11.78 \mathrm{~min}$. $[\alpha]_{\mathrm{D}}^{20}$ $-49.1\left(c=1.12, \mathrm{CHCl}_{3}\right)(96 \%$ ee $) ;{ }^{1} \mathrm{H}$ NMR $\left(300 \mathrm{MHz}, \mathrm{CDCl}_{3}\right) \delta$ 8.11 (bs, $1 \mathrm{H}), 7.36$ (d, $J=8.6 \mathrm{~Hz}, 2 \mathrm{H}), 7.27$ (d, $J=8.5 \mathrm{~Hz}, 2 \mathrm{H}), 7.15$ $(\mathrm{dd}, J=3.2,2.5 \mathrm{~Hz}, 1 \mathrm{H}), 7.12(\mathrm{~d}, J=8.6 \mathrm{~Hz}, 1 \mathrm{H}), 6.61(\mathrm{~d}, J=8.6 \mathrm{~Hz}$, $1 \mathrm{H}), 6.48$ (ddd, $J=3.0,2.0,0.8 \mathrm{~Hz}, 1 \mathrm{H}), 5.42-5.32(\mathrm{~m}, 2 \mathrm{H}), 5.29-$ $5.18(\mathrm{~m}, 1 \mathrm{H}), 4.77(\mathrm{bs}, 1 \mathrm{H}) ;{ }^{13} \mathrm{C} \mathrm{NMR}\left(75 \mathrm{MHz}, \mathrm{CDCl}_{3}\right) \delta 146.7$ (C), $138.8(\mathrm{C}), 131.7(\mathrm{CH}), 131.2(\mathrm{C}), 129.4(\mathrm{CH}), 128.2(\mathrm{C}), 125.6$ $(\mathrm{CH}), 120.9(\mathrm{C}), 115.0(\mathrm{C}), 112.9(\mathrm{CH}), 111.3(\mathrm{CH}), 100.6(\mathrm{CH})$, $77.7\left(\mathrm{CH}_{2}\right), 42.9(\mathrm{CH})$; HRMS (ESI) $\mathrm{m} / z:[\mathrm{M}+\mathrm{H}]^{+}$calcd for $\mathrm{C}_{16} \mathrm{H}_{14} \mathrm{~N}_{2} \mathrm{O}_{3} \mathrm{Br}$, 361.0182; found, 361.0195 .

(S)-4-(1-(4-Methoxyphenyl)-2-nitroethyl)-1H-indol-5-ol (3bd). The product $3 \mathbf{b d}$ was obtained as a brown solid $(22.5 \mathrm{mg}, 0.072$ mmol, $72 \%$ yield); mp $128-132{ }^{\circ} \mathrm{C}$. Enantiomeric excess (94\%) was determined by chiral HPLC (Chiralpak AD-H), hexane- ${ }^{i} \mathrm{PrOH}$ 80:20, $1.0 \mathrm{~mL} / \mathrm{min}$, major enantiomer $t_{\mathrm{r}}=19.50 \mathrm{~min}$, minor enantiomer $t_{\mathrm{r}}=$ 22.35 min. $[\alpha]_{\mathrm{D}}^{20}-11.3(c=0.67, \mathrm{MeOH})(94 \%$ ee $) ;{ }^{1} \mathrm{H}$ NMR $(300$ $\left.\mathrm{MHz}, \mathrm{CDCl}_{3}\right) \delta 8.08(\mathrm{bs}, 1 \mathrm{H}), 7.32(\mathrm{~d}, J=8.6 \mathrm{~Hz}, 2 \mathrm{H}), 7.20-7.09$ $(\mathrm{m}, 2 \mathrm{H}), 6.80(\mathrm{~d}, J=8.9 \mathrm{~Hz}, 2 \mathrm{H}), 6.64(\mathrm{~d}, J=8.5 \mathrm{~Hz}, 1 \mathrm{H}), 6.52$ (ddd, $J=3.0,2.0,0.9 \mathrm{~Hz}, 1 \mathrm{H}), 5.45-5.16(\mathrm{~m}, 3 \mathrm{H}), 4.63(\mathrm{bs}, 1 \mathrm{H}), 3.73(\mathrm{~s}$, $3 \mathrm{H}) ;{ }^{13} \mathrm{C}$ NMR $\left(75 \mathrm{MHz}, \mathrm{CDCl}_{3}\right) \delta 158.5(\mathrm{C}), 146.8(\mathrm{C}), 131.6(\mathrm{C})$, $131.3(\mathrm{C}), 128.6(\mathrm{CH}), 125.4(\mathrm{CH}), 116.0(\mathrm{C}), 114.1(\mathrm{CH}), 113.2$ $(\mathrm{CH}), 111.0(\mathrm{CH}), 100.9(\mathrm{CH}), 78.1\left(\mathrm{CH}_{2}\right), 55.2\left(\mathrm{CH}_{3}\right), 42.7(\mathrm{CH})$; HRMS (ESI) $m / z:[\mathrm{M}+\mathrm{H}]^{+}$calcd for $\mathrm{C}_{17} \mathrm{H}_{17} \mathrm{~N}_{2} \mathrm{O}_{4}, 313.1183$; found, 313.1193.

(S)-4-(1-(Furan-2-yl)-2-nitroethyl)-1H-indol-5-ol (3bi). The product 3bi was obtained as a colorless oil $(22.6 \mathrm{mg}, 0.083 \mathrm{mmol}, 83 \%$ yield). Enantiomeric excess (95\%) was determined by chiral HPLC (Chiralpak AD-H), hexane- ${ }^{i} \mathrm{PrOH}$ 80:20, $1.0 \mathrm{~mL} / \mathrm{min}$, major enantiomer $t_{\mathrm{r}}=19.99 \mathrm{~min}$, minor enantiomer $t_{\mathrm{r}}=16.94 \mathrm{~min}$. $[\alpha]_{\mathrm{D}}^{20}$ $-32.11\left(c=1.06, \mathrm{CHCl}_{3}\right)(95 \% \mathrm{ee}) ;{ }^{1} \mathrm{H} \mathrm{NMR}\left(300 \mathrm{MHz}, \mathrm{CDCl}_{3}\right) \delta$ $8.15(\mathrm{bs}, 1 \mathrm{H}), 7.36-7.34(\mathrm{~m}, 1 \mathrm{H}), 7.21-7.15(\mathrm{~m}, 2 \mathrm{H}), 6.71(\mathrm{~d}, J=8.6$ $\mathrm{Hz}, 1 \mathrm{H}), 6.39$ (ddd, $J=3.1,2.1,0.9 \mathrm{~Hz}, 1 \mathrm{H}), 6.31(\mathrm{dd}, J=3.3,1.9 \mathrm{~Hz}$, $1 \mathrm{H}), 6.16(\mathrm{dt}, J=3.3,0.9 \mathrm{~Hz}, 1 \mathrm{H}), 5.62(\mathrm{t}, J=8.0 \mathrm{~Hz}, 1 \mathrm{H}), 5.33(\mathrm{dd}, J$ $=13.1,8.2 \mathrm{~Hz}, 1 \mathrm{H}), 5.07(\mathrm{dd}, J=13.0,7.0 \mathrm{~Hz}, 1 \mathrm{H}), 5.04(\mathrm{bs}, 1 \mathrm{H}) ;{ }^{13} \mathrm{C}$ NMR (75.5 MHz, $\left.\mathrm{CDCl}_{3}\right) \delta 152.3(\mathrm{C}), 147.1(\mathrm{C}), 141.9(\mathrm{CH}), 131.4$ (C), $127.9(\mathrm{C}), 125.5(\mathrm{CH}), 113.1(\mathrm{CH}), 113.0(\mathrm{C}), 111.6(\mathrm{CH})$, $110.6(\mathrm{CH}), 107.0(\mathrm{CH}), 100.6(\mathrm{CH}), 76.1\left(\mathrm{CH}_{2}\right), 37.1(\mathrm{CH})$; HRMS (ESI) $m / z:[\mathrm{M}+\mathrm{H}]^{+}$calcd for $\mathrm{C}_{14} \mathrm{H}_{13} \mathrm{~N}_{2} \mathrm{O}_{4}, 273.0870$; found, 273.0876.

(S)-4-(2-Nitro-1-(thiophen-2-yl)ethyl)-1H-indol-5-ol (3bj). The product $3 \mathbf{b j}$ was obtained as a colorless oil $(28.4 \mathrm{mg}, 0.098 \mathrm{mmol}$, $98 \%$ yield). Enantiomeric excess (95\%) was determined by chiral HPLC (Chiralpak AD-H), hexane- $-^{i}$ PrOH 80:20, $1.0 \mathrm{~mL} / \mathrm{min}$, major enantiomer $t_{\mathrm{r}}=24.18 \mathrm{~min}$, minor enantiomer $t_{\mathrm{r}}=17.67 \mathrm{~min}$. $[\alpha]_{\mathrm{D}}^{20}$ $-10.61\left(c=0.15, \mathrm{CHCl}_{3}\right)(95 \% \mathrm{ee}) ;{ }^{1} \mathrm{H} \mathrm{NMR}\left(300 \mathrm{MHz}, \mathrm{CDCl}_{3}\right) \delta$ $8.12(\mathrm{bs}, 1 \mathrm{H}), 7.20-7.17(\mathrm{~m}, 2 \mathrm{H}), 7.15(\mathrm{t}, J=1.0 \mathrm{~Hz}, 1 \mathrm{H}), 7.02(\mathrm{dt}, J$ $=3.5,1.1 \mathrm{~Hz}, 1 \mathrm{H}), 6.91(\mathrm{dd}, J=5.1,3.6 \mathrm{~Hz}, 1 \mathrm{H}), 6.67(\mathrm{~d}, J=8.6 \mathrm{~Hz}$, $1 \mathrm{H}), 6.52(\mathrm{ddd}, J=3.1,2.1,0.9 \mathrm{~Hz}, 1 \mathrm{H}), 5.72(\mathrm{td}, J=7.7,0.9 \mathrm{~Hz}, 1 \mathrm{H})$,

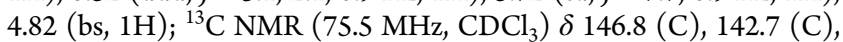
$131.3(\mathrm{C}), 128.0(\mathrm{C}), 126.7(\mathrm{CH}), 125.5(\mathrm{CH}), 124.9(\mathrm{CH}), 124.7$ $(\mathrm{CH}), 115.1(\mathrm{C}), 113.0(\mathrm{CH}), 111.5(\mathrm{CH}), 100.6(\mathrm{CH}), 78.4\left(\mathrm{CH}_{2}\right)$, $39.0(\mathrm{CH})$; HRMS (ESI) $m / z:[\mathrm{M}+\mathrm{H}]^{+}$calcd for $\mathrm{C}_{14} \mathrm{H}_{13} \mathrm{~N}_{2} \mathrm{O}_{3} \mathrm{~S}$, 289.0641; found, 289.0650 .

(S,E)-4-(1-Nitro-4-phenylbut-3-en-2-yl)-1H-indol-5-ol (3bk). The product 3bk was obtained as a colorless oil $(29.3 \mathrm{mg}, 0.095 \mathrm{mmol}$, $95 \%$ yield). Enantiomeric excess $(89 \%)$ was determined by chiral HPLC (Chiralpak AS-H), hexane- ${ }^{i} \mathrm{PrOH} 80: 20,1.0 \mathrm{~mL} / \mathrm{min}$, major enantiomer $t_{\mathrm{r}}=66.76 \mathrm{~min}$, minor enantiomer $t_{\mathrm{r}}=78.59 \mathrm{~min}$. $[\alpha]_{\mathrm{D}}^{20}$ -66.12 (c = 0.80, $\left.\mathrm{CHCl}_{3}\right)\left(89 \%\right.$ ee); ${ }^{1} \mathrm{H} \mathrm{NMR}\left(300 \mathrm{MHz}, \mathrm{CDCl}_{3}\right) \delta$ $8.13(\mathrm{~s}, 1 \mathrm{H}), 7.37-7.09(\mathrm{~m}, 7 \mathrm{H}), 6.80-6.45(\mathrm{~m}, 5 \mathrm{H}), 5.14(\mathrm{dd}, J=$ $14.1,10.9 \mathrm{~Hz}, 1 \mathrm{H}), 4.95(\mathrm{dt}, J=6.5,5.0 \mathrm{~Hz}, 2 \mathrm{H}), 4.84(\mathrm{~s}, 1 \mathrm{H}) ;{ }^{13} \mathrm{C}$ NMR (75.5 MHz, $\left.\mathrm{CDCl}_{3}\right) \delta 146.9(\mathrm{C}), 136.7(\mathrm{C}), 132.4(\mathrm{CH}), 131.4$ (C), $128.4(\mathrm{CH}), 127.9(\mathrm{C}), 127.6(\mathrm{CH}), 126.4(\mathrm{CH}), 126.4(\mathrm{CH})$, $125.5(\mathrm{CH}), 114.2(\mathrm{C}), 112.8(\mathrm{CH}), 111.1(\mathrm{CH}), 100.7(\mathrm{CH}), 77.9$
$\left(\mathrm{CH}_{2}\right), 42.2(\mathrm{CH})$; HRMS (ESI) $m / z:[\mathrm{M}+\mathrm{H}]^{+}$calcd for $\mathrm{C}_{18} \mathrm{H}_{17} \mathrm{~N}_{2} \mathrm{O}_{3}, 309.1234$; found, 309.1247 .

(S)-4-(1-Nitro-4-phenylbutan-2-yl)-1H-indol-5-ol (3bl). The product $3 \mathrm{bl}$ was obtained as a colorless oil $(13.8 \mathrm{mg}, 0.044 \mathrm{mmol}, 44 \%$ yield). Enantiomeric excess (87\%) was determined by chiral HPLC (Chiralpak AD-H), hexane- ${ }^{i} \mathrm{PrOH} 80: 20,1.0 \mathrm{~mL} / \mathrm{min}$, major enantiomer $t_{\mathrm{r}}=12.79 \mathrm{~min}$, minor enantiomer $t_{\mathrm{r}}=10.96 \mathrm{~min}$. $[\alpha]_{\mathrm{D}}^{20}$ $-13.53\left(c=0.67, \mathrm{CHCl}_{3}\right)(87 \%$ ee $) ;{ }^{1} \mathrm{H} \mathrm{NMR}\left(300 \mathrm{MHz}, \mathrm{CDCl}_{3}\right) \delta$ 8.10 (bs, $1 \mathrm{H}), 7.25-7.12(\mathrm{~m}, 5 \mathrm{H}), 7.12-7.04(\mathrm{~m}, 2 \mathrm{H}), 6.67(\mathrm{~d}, J=8.6$ $\mathrm{Hz}, 1 \mathrm{H}), 6.50$ (ddd, $J=3.1,2.0,0.9 \mathrm{~Hz}, 1 \mathrm{H}), 5.03-4.83(\mathrm{~m}, 2 \mathrm{H}), 4.66$ (bs, $1 \mathrm{H}), 4.21-4.08(\mathrm{~m}, 1 \mathrm{H}), 2.57-2.45(\mathrm{~m}, 2 \mathrm{H}), 2.19-2.00(\mathrm{~m}$, $2 \mathrm{H}) ;{ }^{13} \mathrm{C}$ NMR $\left(75.5 \mathrm{MHz}, \mathrm{CDCl}_{3}\right) \delta 147.3(\mathrm{C}), 141.7(\mathrm{C}), 131.3$ (C), $128.3(\mathrm{CH}), 128.3(\mathrm{CH}), 125.8(\mathrm{CH}), 125.2(\mathrm{CH}), 114.9(\mathrm{C})$, $112.8(\mathrm{CH}), 110.8(\mathrm{CH}), 101.0(\mathrm{CH}), 78.7\left(\mathrm{CH}_{2}\right), 33.6\left(\mathrm{CH}_{2}\right), 32.6$ $\left(\mathrm{CH}_{2}\right), 30.3(\mathrm{CH})$; HRMS (ESI) $m / z:[\mathrm{M}+\mathrm{H}]^{+}$calcd for $\mathrm{C}_{18} \mathrm{H}_{19} \mathrm{~N}_{2} \mathrm{O}_{3}, 311.1390$; found, 311.1397 .

(S)-1-Methyl-4-(2-nitro-1-phenylethyl)-1H-indol-5-ol (3ca). The product 3ca was obtained as a colorless oil $(26.1 \mathrm{mg}, 0.088 \mathrm{mmol}$, $88 \%$ yield). Enantiomeric excess (96\%) was determined by chiral HPLC (Chiralpak IC), hexane $-{ }^{i} \mathrm{PrOH} 80: 20,1.0 \mathrm{~mL} / \mathrm{min}$, major enantiomer $t_{\mathrm{r}}=7.17 \mathrm{~min}$, minor enantiomer $t_{\mathrm{r}}=6.50 \mathrm{~min} .[\alpha]_{\mathrm{D}}^{20}-48.4$ $\left(c=1.30, \mathrm{CHCl}_{3}\right)(96 \%$ ee $) ;{ }^{1} \mathrm{H}$ NMR $\left(300 \mathrm{MHz}, \mathrm{CDCl}_{3}\right) \delta 7.43-$ $7.36(\mathrm{~m}, 2 \mathrm{H}), 7.34-7.15(\mathrm{~m}, 4 \mathrm{H}), 7.08(\mathrm{~d}, J=8.6 \mathrm{~Hz}, 1 \mathrm{H}), 7.02(\mathrm{~d}, J$ $=3.1 \mathrm{~Hz}, 1 \mathrm{H}), 6.67(\mathrm{~d}, J=8.6 \mathrm{~Hz}, 1 \mathrm{H}), 6.44(\mathrm{~d}, J=2.6 \mathrm{~Hz}, 1 \mathrm{H})$, $5.51-5.23(\mathrm{~m}, 3 \mathrm{H}), 4.58(\mathrm{~s}, 1 \mathrm{H}), 3.71(\mathrm{~s}, 3 \mathrm{H}) ;{ }^{13} \mathrm{C} \mathrm{NMR}(75.5 \mathrm{MHz}$, $\left.\mathrm{CDCl}_{3}\right) \delta 146.6(\mathrm{C}), 139.6(\mathrm{C}), 132.4(\mathrm{C}), 129.9(\mathrm{CH}), 128.9(\mathrm{C})$, $128.7(\mathrm{CH}), 127.5(\mathrm{CH}), 127.0(\mathrm{CH}), 115.8(\mathrm{C}), 112.7(\mathrm{CH}), 109.4$ $(\mathrm{CH}), 98.9(\mathrm{CH}), 77.8\left(\mathrm{CH}_{2}\right), 43.2(\mathrm{CH}), 33.0\left(\mathrm{CH}_{3}\right)$; HRMS (ESI) $m / z:[\mathrm{M}+\mathrm{H}]^{+}$calcd for $\mathrm{C}_{17} \mathrm{H}_{17} \mathrm{~N}_{2} \mathrm{O}_{3}, 297.1234$; found, 297.1239.

(S)-2-Methyl-4-(2-nitro-1-phenylethyl)-1H-indol-5-ol (3da). The product 3da was obtained as a colorless oil $(27.1 \mathrm{mg}$, inseparable mixture of $\mathrm{C}-4$ alkylated product:C-3 alkylated product $\left(5.4: 1\right.$ by ${ }^{1} \mathrm{H}$ NMR), $80 \%$ yield). Enantiomeric excess (96\%) was determined by chiral HPLC (Chiralcel OD-H), hexane- ${ }^{i} \mathrm{PrOH} 80: 20,1.0 \mathrm{~mL} / \mathrm{min}$, major enantiomer $t_{\mathrm{r}}=13.47 \mathrm{~min}$, minor enantiomer $t_{\mathrm{r}}=11.02 \mathrm{~min}$. $[\alpha]_{\mathrm{D}}^{20}-64.1\left(c=1.43, \mathrm{CHCl}_{3}\right)(96 \%$ ee $) ;{ }^{1} \mathrm{H}$ NMR $(300 \mathrm{MHz}$, $\left.\mathrm{CDCl}_{3}\right) \delta 7.74(\mathrm{~s}, 1 \mathrm{H}), 7.37(\mathrm{~d}, J=7.2 \mathrm{~Hz}, 2 \mathrm{H}), 7.22(\mathrm{t}, J=3.7 \mathrm{~Hz}$, $3 \mathrm{H}), 6.96(\mathrm{~d}, J=8.5 \mathrm{~Hz}, 1 \mathrm{H}), 6.49(\mathrm{~d}, J=8.5 \mathrm{~Hz}, 1 \mathrm{H}), 6.17(\mathrm{~s}, 1 \mathrm{H})$, 5.45-5.19 (m, 3H), $4.60(\mathrm{~s}, 1 \mathrm{H}), 2.33(\mathrm{~s}, 3 \mathrm{H}) ;{ }^{13} \mathrm{C}$ NMR $(75.5 \mathrm{MHz}$, $\left.\mathrm{CDCl}_{3}\right) \delta 146.7$ (C), 139.7 (C), 136.5 (C), 131.4 (C), 129.5 (C), $128.6(\mathrm{CH}), 127.5(\mathrm{CH}), 127.0(\mathrm{CH}), 114.9(\mathrm{C}), 111.5(\mathrm{CH}), 110.2$ $(\mathrm{CH}), 98.8(\mathrm{CH}), 77.9\left(\mathrm{CH}_{2}\right), 43.3(\mathrm{CH}), 13.8\left(\mathrm{CH}_{3}\right)$. HRMS (ESI) $m / z:[\mathrm{M}+\mathrm{H}]^{+}$calcd for $\mathrm{C}_{17} \mathrm{H}_{17} \mathrm{~N}_{2} \mathrm{O}_{3}, 297.1234$; found, 297.1242.

Scope of the Reaction with 6-Hydroxyindole. (S)-7-(1-Phenyl2-nitroethyl)-1H-indol-6-ol (3ea). The product 3ea was obtained as a dark oil ( $17.6 \mathrm{mg}, 0.062 \mathrm{mmol}, 62 \%$ yield). Enantiomeric excess (95\%) was determined by chiral HPLC (Chiralpak AS-H), hexane $-{ }^{i} \mathrm{PrOH}$ $90: 10,1.0 \mathrm{~mL} / \mathrm{min}$, major enantiomer $t_{\mathrm{r}}=42.21 \mathrm{~min}$, minor enantiomer $t_{\mathrm{r}}=49.20 \mathrm{~min}$. $[\alpha]_{\mathrm{D}}^{20}-67.5\left(c=0.92, \mathrm{CHCl}_{3}\right)(95 \%$ ee); ${ }^{1} \mathrm{H}$ NMR $\left(300 \mathrm{MHz}, \mathrm{CDCl}_{3}\right) \delta 7.73(\mathrm{~s}, 1 \mathrm{H}), 7.41-7.25(\mathrm{~m}, 6 \mathrm{H})$, $6.94(\mathrm{dd}, J=3.3,2.4 \mathrm{~Hz}, 1 \mathrm{H}), 6.59(\mathrm{~d}, J=8.4 \mathrm{~Hz}, 1 \mathrm{H}), 6.41(\mathrm{dd}, J=$ 3.3, $2.0 \mathrm{~Hz}, 1 \mathrm{H}), 5.45-5.37(\mathrm{~m}, 1 \mathrm{H}), 5.35-5.17(\mathrm{~m}, 2 \mathrm{H}), 4.92(\mathrm{~s}$, $1 \mathrm{H}) ;{ }^{13} \mathrm{C} \mathrm{NMR}\left(75 \mathrm{MHz}, \mathrm{CDCl}_{3}\right) \delta 149.0(\mathrm{C}), 138.7$ (C), 135.7 (C), $129.1(\mathrm{CH}), 127.6(\mathrm{CH}), 127.4(\mathrm{CH}), 123.7(\mathrm{CH}), 123.3(\mathrm{C}), 120.8$ $(\mathrm{CH}), 110.7(\mathrm{CH}), 107.6(\mathrm{C}), 102.9(\mathrm{CH}), 77.2\left(\mathrm{CH}_{2}\right), 41.3(\mathrm{CH})$; HRMS (ESI) $m / z:[\mathrm{M}+\mathrm{H}]^{+}$calcd for $\mathrm{C}_{16} \mathrm{H}_{15} \mathrm{~N}_{2} \mathrm{O}_{3}$, 283.1077; found, 283.1088.

(S)-7-(1-(4-Bromophenyl)-2-nitroethyl)-1H-indol-6-ol (3eb). The product 3eb was obtained as a yellow solid $(24.9 \mathrm{mg}, 0.069 \mathrm{mmol}$, $69 \%$ yield); mp $190-194{ }^{\circ} \mathrm{C}$. Enantiomeric excess (84\%; 99\% after crystallization) was determined by chiral HPLC (Chiralpak AD-H), hexane $-{ }^{i} \mathrm{PrOH} 80: 20,1.0 \mathrm{~mL} / \mathrm{min}$, major enantiomer $t_{\mathrm{r}}=8.97 \mathrm{~min}$, minor enantiomer $t_{\mathrm{r}}=10.10 \mathrm{~min} .[\alpha]_{\mathrm{D}}^{20}-0.17\left(c=1.085, \mathrm{CHCl}_{3}\right)$ (84\% ee); ${ }^{1} \mathrm{H}$ NMR (300 MHz, $\left.\mathrm{CD}_{3} \mathrm{OD}\right) \delta 7.50-7.36(\mathrm{~m}, 4 \mathrm{H}), 7.30$ $(\mathrm{d}, J=8.4 \mathrm{~Hz}, 1 \mathrm{H}), 7.09(\mathrm{~d}, J=3.2 \mathrm{~Hz}, 1 \mathrm{H}), 6.64(\mathrm{~d}, J=8.4 \mathrm{~Hz}, 1 \mathrm{H})$, $6.36(\mathrm{~d}, J=3.2 \mathrm{~Hz}, 1 \mathrm{H}), 5.53-5.39(\mathrm{~m}, 2 \mathrm{H}), 5.34(\mathrm{dd}, J=11.5,3.7$ $\mathrm{Hz}, 1 \mathrm{H}) ;{ }^{13} \mathrm{C}$ NMR $\left(75 \mathrm{MHz}, \mathrm{CD}_{3} \mathrm{OD}\right) \delta 152.7$ (C), 141.7 (C), 138.3 (C), $133.2(\mathrm{CH}), 131.9(\mathrm{CH}), 125.2(\mathrm{CH}), 124.6(\mathrm{C}), 122.4(\mathrm{C})$, $122.0(\mathrm{C}), 112.1(\mathrm{C}), 109.3(\mathrm{C}), 103.7(\mathrm{CH}), 79.7\left(\mathrm{CH}_{2}\right), 43.8(\mathrm{CH})$; 
HRMS (ESI) $m / z$ : $[\mathrm{M}+\mathrm{H}]^{+}$calcd for $\mathrm{C}_{16} \mathrm{H}_{14} \mathrm{~N}_{2} \mathrm{O}_{3} \mathrm{Br}$, 361.0182; found, 361.0199 .

(S)-7-(1-(4-(Trifluoromethyl)phenyl)-2-nitroethyl)-1H-indol-6-ol (3ec). The product $3 \mathrm{ec}$ was obtained as a brown solid $(27.0 \mathrm{mg}, 0.077$ mmol, 77\% yield); mp 152-157. Enantiomeric excess (88\%) was determined by chiral HPLC (Chiralpak AD-H), hexane- ${ }^{i} \mathrm{PrOH}$ 80:20, $1.0 \mathrm{~mL} / \mathrm{min}$, major enantiomer $t_{\mathrm{r}}=6.58 \mathrm{~min}$, minor enantiomer $t_{\mathrm{r}}=$ $7.50 \mathrm{~min} .[\alpha]_{\mathrm{D}}^{20}-46.7\left(c=0.85, \mathrm{CHCl}_{3}\right)(88 \%$ ee $) ;{ }^{1} \mathrm{H}$ NMR $(300$ $\left.\mathrm{MHz}, \mathrm{CDCl}_{3}\right) \delta 7.92(\mathrm{~s}, 1 \mathrm{H}), 7.55(\mathrm{~d}, J=8.4 \mathrm{~Hz}, 2 \mathrm{H}), 7.48(\mathrm{~d}, J=8.3$ $\mathrm{Hz}, 2 \mathrm{H}), 7.40(\mathrm{~d}, J=8.3 \mathrm{~Hz}, 1 \mathrm{H}), 7.04(\mathrm{dd}, J=3.3,2.4 \mathrm{~Hz}, 1 \mathrm{H}), 6.59$ $(\mathrm{d}, J=8.4 \mathrm{~Hz}, 1 \mathrm{H}), 6.45(\mathrm{dd}, J=3.3,1.9 \mathrm{~Hz}, 1 \mathrm{H}), 5.41-5.19(\mathrm{~m}, 3 \mathrm{H})$, $4.89(\mathrm{~s}, 1 \mathrm{H}) ;{ }^{13} \mathrm{C}$ NMR $\left(75 \mathrm{MHz}, \mathrm{CDCl}_{3}\right) \delta 149.1$ (C), $143.0(\mathrm{C})$, $135.6(\mathrm{C}), 129.8\left(J_{\mathrm{C}-\mathrm{F}}=32.3 \mathrm{~Hz}, \mathrm{C}\right), 127.9(\mathrm{CH}), 125.9\left(J_{\mathrm{C}-\mathrm{F}}=3.5\right.$ $\mathrm{Hz}, \mathrm{C}), 123.89(\mathrm{CH}), 123.88\left(\mathrm{~d}, J_{\mathrm{C}-\mathrm{F}}=272.0 \mathrm{~Hz}, \mathrm{CF}_{3}\right), 123.3(\mathrm{C})$, $121.3(\mathrm{CH}), 110.7(\mathrm{CH}), 106.6(\mathrm{C}), 103.4(\mathrm{CH}), 77.12\left(\mathrm{CH}_{2}\right), 41.5$ $(\mathrm{CH}) ;{ }^{19} \mathrm{~F}$ NMR $\left(282.4 \mathrm{MHz}, \mathrm{CDCl}_{3}\right) \delta-63.11\left(\mathrm{~s}, \mathrm{CF}_{3}\right)$; HRMS (ESI) $m / z:[\mathrm{M}+\mathrm{H}]^{+}$calcd for $\mathrm{C}_{17} \mathrm{H}_{13} \mathrm{~N}_{2} \mathrm{O}_{3} \mathrm{~F}_{3}, 351.0951$; found, 351.0953.

(S)-7-(1-(4-Hydroxy-3-methoxyphenyl)-2-nitroethyl)-1H-indol-6ol (3eh). The product $3 \mathrm{eh}$ was obtained as a dark oil $(12.2 \mathrm{mg}, 0.037$ mmol, $37 \%$ yield). Enantiomeric excess (90\%) was determined by chiral HPLC (Chiralpak IC), hexane- ${ }^{i} \mathrm{PrOH} 70: 30,1.0 \mathrm{~mL} / \mathrm{min}$, major enantiomer $t_{\mathrm{r}}=7.78 \mathrm{~min}$, minor enantiomer $t_{\mathrm{r}}=5.26 \mathrm{~min}$. $[\alpha]_{\mathrm{D}}^{20}$ $-48.7\left(c=0.33, \mathrm{CHCl}_{3}\right)(90 \%$ ee $) ;{ }^{1} \mathrm{H}$ NMR $\left(300 \mathrm{MHz}, \mathrm{CDCl}_{3}\right) \delta$ $7.69(\mathrm{~s}, 1 \mathrm{H}), 7.38(\mathrm{~d}, J=8.4 \mathrm{~Hz}, 1 \mathrm{H}), 6.96-6.91(\mathrm{~m}, 2 \mathrm{H}), 6.89(\mathrm{~d}, J=$ $8.1 \mathrm{~Hz}, 1 \mathrm{H}), 6.85(\mathrm{~d}, J=2.0 \mathrm{~Hz}, 1 \mathrm{H}), 6.62(\mathrm{~d}, J=8.4 \mathrm{~Hz}, 1 \mathrm{H}), 6.39$ $(\mathrm{dd}, J=3.3,2.0 \mathrm{~Hz}, 1 \mathrm{H}), 5.58(\mathrm{~s}, 1 \mathrm{H}), 5.39(\mathrm{dd}, J=8.9,6.4 \mathrm{~Hz}, 1 \mathrm{H})$, $5.22(\mathrm{dd}, J=13.3,8.9 \mathrm{~Hz}, 1 \mathrm{H}), 5.12(\mathrm{dd}, J=13.2,6.5 \mathrm{~Hz}, 1 \mathrm{H}), 4.93$ (s, 1H), $3.78(\mathrm{~s}, 3 \mathrm{H}) ;{ }^{13} \mathrm{C} \mathrm{NMR}\left(75 \mathrm{MHz}, \mathrm{CDCl}_{3}\right) \delta 149.2(\mathrm{C}), 147.7$ (C), 145.7 (C), 136.1 (C), 130.9 (C), $124.1(\mathrm{CH}), 123.9$ (C), 121.2 (CH), $119.7(\mathrm{CH}), 115.1(\mathrm{CH}), 111.1(\mathrm{CH}), 110.9(\mathrm{CH}), 103.2$ $(\mathrm{CH}), 77.7\left(\mathrm{CH}_{2}\right), 56.4\left(\mathrm{CH}_{3}\right), 41.4(\mathrm{CH})$; HRMS (ESI) $m / z:[\mathrm{M}+$ $\mathrm{H}]^{+}$calcd for $\mathrm{C}_{17} \mathrm{H}_{16} \mathrm{~N}_{2} \mathrm{O}_{5}, 329.1132$; found, 329.1129 .

(S)-7-(1-(Furan-2-yl)-2-nitroethyl)-1H-indol-6-ol (3ei). The product 3ei was obtained as a colorless oil $(26.9 \mathrm{mg}, 0.099 \mathrm{mmol}$, $99 \%$ yield). Enantiomeric excess (94\%) was determined by chiral HPLC (Chiralpak AD-H), hexane- ${ }^{i} \mathrm{PrOH} 90: 10,1.0 \mathrm{~mL} / \mathrm{min}$, major enantiomer $t_{\mathrm{r}}=25.78 \mathrm{~min}$, minor enantiomer $t_{\mathrm{r}}=28.51 \mathrm{~min}$. $[\alpha]_{\mathrm{D}}^{20}$ $-45.8\left(c=1.05, \mathrm{CHCl}_{3}\right)(94 \% \mathrm{ee}) ;{ }^{1} \mathrm{H}$ NMR $\left(300 \mathrm{MHz}, \mathrm{CDCl}_{3}\right) \delta$ $8.03(\mathrm{bs}, 1 \mathrm{H}), 7.43(\mathrm{t}, J=0.9 \mathrm{~Hz}, 1 \mathrm{H}), 7.41(\mathrm{dd}, J=5.4,0.7 \mathrm{~Hz}, 1 \mathrm{H})$, $7.02(\mathrm{dd}, J=3.2,2.4 \mathrm{~Hz}, 1 \mathrm{H}), 6.63(\mathrm{~d}, J=8.4 \mathrm{~Hz}, 1 \mathrm{H}), 6.45(\mathrm{dd}, J=$ $3.3,2.0 \mathrm{~Hz}, 1 \mathrm{H}), 6.39(\mathrm{dd}, J=3.3,1.9 \mathrm{~Hz}, 1 \mathrm{H}), 6.31(\mathrm{dt}, J=3.3,0.9$ $\mathrm{Hz}, 1 \mathrm{H}), 5.65(\mathrm{ddd}, J=9.2,6.1,0.6 \mathrm{~Hz}, 1 \mathrm{H}), 5.22(\mathrm{dd}, J=13.2,9.2$ $\mathrm{Hz}, 1 \mathrm{H}), 5.08(\mathrm{bs}, 1 \mathrm{H}), 4.97(\mathrm{dd}, J=13.2,6.1 \mathrm{~Hz}, 1 \mathrm{H}) ;{ }^{13} \mathrm{C}$ NMR $\left(75.5 \mathrm{MHz}, \mathrm{CDCl}_{3}\right) \delta 151.8(\mathrm{C}), 148.8(\mathrm{C}), 142.3(\mathrm{CH}), 135.3(\mathrm{C})$ $123.8(\mathrm{CH}), 123.5(\mathrm{C}), 121.1(\mathrm{CH}), 111.0(\mathrm{CH}), 110.3(\mathrm{CH}), 107.7$ $(\mathrm{CH}), 105.3(\mathrm{C}), 102.9(\mathrm{CH}), 75.4\left(\mathrm{CH}_{2}\right), 35.1(\mathrm{CH})$; HRMS (ESI) $m / z:[\mathrm{M}+\mathrm{H}]^{+}$calcd for $\mathrm{C}_{14} \mathrm{H}_{13} \mathrm{~N}_{2} \mathrm{O}_{4}, 273.0870$; found, 273.0877.

(S)-7-(2-Nitro-1-(thiophen-2-yl)ethyl)-1H-indol-6-ol (3ej). The product 3ej was obtained as a colorless oil $(26.6 \mathrm{mg}, 0.092 \mathrm{mmol}$, $92 \%$ yield). Enantiomeric excess (94\%) was determined by chiral HPLC (Chiralpak AD-H), hexane- $-^{i}$ PrOH 80:20, $1.0 \mathrm{~mL} / \mathrm{min}$, major enantiomer $t_{\mathrm{r}}=11.71 \mathrm{~min}$, minor enantiomer $t_{\mathrm{r}}=10.25 \mathrm{~min}$. $[\alpha]_{\mathrm{D}}^{20}$ $-8.84\left(c=0.91, \mathrm{CHCl}_{3}\right)(94 \%$ ee $) ;{ }^{1} \mathrm{H}$ NMR $\left(300 \mathrm{MHz}, \mathrm{CDCl}_{3}\right) \delta$ $7.89(\mathrm{bs}, 1 \mathrm{H}), 7.42(\mathrm{dd}, J=8.4,0.6 \mathrm{~Hz}, 1 \mathrm{H}), 7.24(\mathrm{dd}, J=5.1,1.2 \mathrm{~Hz}$, $1 \mathrm{H}), 7.06(\mathrm{dt}, J=3.5,1.2 \mathrm{~Hz}, 1 \mathrm{H}), 7.00(\mathrm{dd}, J=3.2,2.4 \mathrm{~Hz}, 1 \mathrm{H}), 6.97$ $(\mathrm{dd}, J=5.1,3.5 \mathrm{~Hz}, 1 \mathrm{H}), 6.62(\mathrm{~d}, J=8.4 \mathrm{~Hz}, 1 \mathrm{H}), 6.45(\mathrm{dd}, J=3.3$, $2.0 \mathrm{~Hz}, 1 \mathrm{H}), 5.73-5.62(\mathrm{~m}, 1 \mathrm{H}), 5.29(\mathrm{dd}, J=13.3,8.3 \mathrm{~Hz}, 1 \mathrm{H}), 5.17$ $(\mathrm{dd}, J=13.3,6.6 \mathrm{~Hz}, 1 \mathrm{H}), 5.07(\mathrm{bs}, 1 \mathrm{H}) ;{ }^{13} \mathrm{C} \mathrm{NMR}(75.5 \mathrm{MHz}$, $\left.\mathrm{CDCl}_{3}\right) \delta 148.8(\mathrm{C}), 142.2(\mathrm{C}), 135.4(\mathrm{C}), 126.9(\mathrm{CH}), 125.5(\mathrm{CH})$, $124.8(\mathrm{CH}), 123.8(\mathrm{CH}), 123.4(\mathrm{C}), 121.2(\mathrm{CH}), 110.6(\mathrm{CH}), 107.1$ (C), $103.0(\mathrm{CH}), 77.7\left(\mathrm{CH}_{2}\right), 37.1(\mathrm{CH})$; HRMS (ESI) $\mathrm{m} / \mathrm{z}:[\mathrm{M}+$ $\mathrm{H}]^{+}$calcd for $\mathrm{C}_{14} \mathrm{H}_{13} \mathrm{~N}_{2} \mathrm{O}_{3} \mathrm{~S}$, 289.0641; found, 289.0650.

(S,E)-7-(1-Nitro-4-phenylbut-3-en-2-yl)-1H-indol-6-ol (3ek). The product 3ek was obtained as a colorless oil $(13.6 \mathrm{mg}, 0.044 \mathrm{mmol}$, $44 \%$ yield). Enantiomeric excess (89\%) was determined by chiral HPLC (Chiralpak AS-H), hexane $-{ }^{i}$ PrOH 80:20, $1.0 \mathrm{~mL} / \mathrm{min}$, major enantiomer $t_{\mathrm{r}}=66.76 \mathrm{~min}$, minor enantiomer $t_{\mathrm{r}}=78.59 \mathrm{~min}$. $[\alpha]_{\mathrm{D}}^{20}$ $-66.12\left(c=0.80, \mathrm{CHCl}_{3}\right)(89 \% \mathrm{ee}) ;{ }^{1} \mathrm{H}$ NMR $\left(300 \mathrm{MHz}, \mathrm{CDCl}_{3}\right) \delta$ $8.25(\mathrm{~s}, 1 \mathrm{H}), 7.41(\mathrm{~d}, J=8.4 \mathrm{~Hz}, 1 \mathrm{H}), 7.38-7.26(\mathrm{~m}, 4 \mathrm{H}), 7.26-7.18$ (m, 1H), 7.13-7.08 (m, 1H), 6.72-6.54 (m, 3H), $6.49(\mathrm{dd}, J=3.2$, $2.0 \mathrm{~Hz}, 1 \mathrm{H}), 5.09(\mathrm{dd}, \mathrm{J}=12.6,8.4 \mathrm{~Hz}, 1 \mathrm{H}), 4.96(\mathrm{dd}, J=12.5,6.4 \mathrm{~Hz}$, $1 \mathrm{H}), 4.93(\mathrm{~s}, 1 \mathrm{H}), 4.82(\mathrm{dt}, J=8.4,6.2 \mathrm{~Hz}, 1 \mathrm{H}) ;{ }^{13} \mathrm{C} \mathrm{NMR}(75.5$ $\left.\mathrm{MHz}, \mathrm{CDCl}_{3}\right) \delta 149.3(\mathrm{C}), 136.3(\mathrm{C}), 135.5(\mathrm{C}), 132.5(\mathrm{CH}), 128.6$ $(\mathrm{CH}), 127.9(\mathrm{CH}), 126.5(\mathrm{CH}), 125.9(\mathrm{CH}), 123.7(\mathrm{CH}), 123.2(\mathrm{C})$, $120.8(\mathrm{CH}), 110.7(\mathrm{CH}), 106.1(\mathrm{C}), 103.2(\mathrm{CH}), 77.4\left(\mathrm{CH}_{2}\right), 40.2$ (CH); HRMS (ESI) $m / z:[\mathrm{M}+\mathrm{H}]^{+}$calcd for $\mathrm{C}_{18} \mathrm{H}_{17} \mathrm{~N}_{2} \mathrm{O}_{3}, 309.1234$; found, 309.1247.

Scope of the Reaction with 7-Hydroxyindole. (S)-6-(1-Phenyl2-nitroethyl)-1H-indol-7-ol (3fa). The product $3 \mathrm{fa}$ was obtained as a dark oil ( $12.7 \mathrm{mg}, 0.045 \mathrm{mmol}, 45 \%$ yield). Enantiomeric excess (18\%) was determined by chiral HPLC (lux-amylose-1), hexane- $-{ }^{i} \mathrm{PrOH}$ $80: 20,1.0 \mathrm{~mL} / \mathrm{min}$, major enantiomer $t_{\mathrm{r}}=11.50 \mathrm{~min}$, minor enantiomer $t_{\mathrm{r}}=12.55 \mathrm{~min}$. $[\alpha]_{\mathrm{D}}^{20}-10.2\left(c=0.26, \mathrm{CHCl}_{3}\right)(18 \%$ ee); ${ }^{1} \mathrm{H}$ NMR $\left(300 \mathrm{MHz}, \mathrm{CDCl}_{3}\right) \delta 8.39(\mathrm{~s}, 1 \mathrm{H}), 7.35-7.25(\mathrm{~m}, 5 \mathrm{H})$, $7.23(\mathrm{~d}, J=8.7 \mathrm{~Hz}, 1 \mathrm{H}), 7.14(\mathrm{~s}, 1 \mathrm{H}), 6.83(\mathrm{~d}, J=8.2 \mathrm{~Hz}, 1 \mathrm{H}), 6.49-$ $6.45(\mathrm{~m}, 1 \mathrm{H}), 5.27(\mathrm{~m}, 2 \mathrm{H}), 5.09(\mathrm{~d}, J=7.9 \mathrm{~Hz}, 2 \mathrm{H}) ;{ }^{13} \mathrm{C}$ NMR $(75$ $\mathrm{MHz}, \mathrm{CDCl}_{3}$ ) $\delta 139.2(\mathrm{C}), 139.1$ (C), $129.3(\mathrm{C}), 129.2(\mathrm{CH}), 127.7$ $(\mathrm{CH}), 127.6(\mathrm{CH}), 127.4(\mathrm{C}), 124.7(\mathrm{CH}), 119.2(\mathrm{CH}), 118.2(\mathrm{C})$, $114.5(\mathrm{CH}), 103.0(\mathrm{CH}), 78.9\left(\mathrm{CH}_{2}\right), 42.6(\mathrm{CH})$; HRMS (ESI) $m / z$ : $[\mathrm{M}+\mathrm{H}]^{+}$calcd for $\mathrm{C}_{16} \mathrm{H}_{14} \mathrm{~N}_{2} \mathrm{O}_{3}$, 283.1077; found, 283.1107 .

Dialkylation Product When 4-Hydroxyindole Is Used. Products $3 \mathbf{a a}^{\prime}$ and $\mathbf{4}$ were obtained as an unseparable mixture. Yet, in some cases, 4 was obtained pure after column chromatography.

5,7-Bis(1-phenyl-2-nitroethyl)-1H-indol-4-ol (4). Dark oil; ${ }^{1} \mathrm{H}$ NMR $\left(300 \mathrm{MHz}, \mathrm{CDCl}_{3}\right) \delta 7.94(\mathrm{~s}, 1 \mathrm{H}), 7.35-7.17(\mathrm{~m}, 10 \mathrm{H})$, $6.99(\mathrm{t}, J=2.9 \mathrm{~Hz}, 1 \mathrm{H}), 6.82(\mathrm{~s}, 0.5 \mathrm{H})^{*}, 6.78(\mathrm{~s}, 0.5 \mathrm{H})^{*}, 6.42(\mathrm{ddd}, J$ $=3.1,2.0,1.0 \mathrm{~Hz}, 1 \mathrm{H}), 5.39-5.29(\mathrm{~m}, 1 \mathrm{H}), 5.22(\mathrm{~s}, 1 \mathrm{H}), 5.22-4.87$ $(\mathrm{m}, 5 \mathrm{H}) ;{ }^{13} \mathrm{C}$ NMR $\left(75 \mathrm{MHz}, \mathrm{CDCl}_{3}\right) \delta 145.9,145.8^{*}, 139.4,139.3^{*}$, $138.2,134.8,129.4,129.3^{*}, 128.9,128.8^{*}, 128.12,128.07^{*}, 127.72$ $127.71 * 127.6,127.5 * 127.4,127.3 *, 124.3,120.7,120.2 *, 118.8$, $115.10,115.06^{*}, 114.9,114.7^{*}, 98.5,78.82,78.81^{*}, 78.4,78.3^{*}, 44.64$, 44.60*, 43.7, 43.3*; HRMS (ESI) $m / z:[\mathrm{M}+\mathrm{H}]^{+}$calcd for $\mathrm{C}_{24} \mathrm{H}_{22} \mathrm{~N}_{3} \mathrm{O}_{5}$, 432.1554; found, 432.1565. “*” indicates the presence of rotamers.

Procedures and Characterization Data for Compounds 6. tert-Butyl (S)-(2-(5-Hydroxy-1H-indol-4-yl)-2-phenylethyl)carbamate (6a). Compound $3 \mathrm{ba}(28.1 \mathrm{mg}, 0.1 \mathrm{mmol})$ and $\mathrm{NiCl}_{2}$. $6 \mathrm{H}_{2} \mathrm{O}(23.8 \mathrm{mg}, 0.1 \mathrm{mmol}, 1$ equiv) were placed in a $10 \mathrm{~mL}$ round bottomed flask, and $\mathrm{MeOH}(1 \mathrm{~mL})$ was added. After the suspension was stirred, $\mathrm{NaBH}_{4}\left(18.9 \mathrm{mg}, 0.5 \mathrm{mmol}, 5\right.$ equiv) was added at $0{ }^{\circ} \mathrm{C}$, and the mixture was stirred at room temperature for $1.5 \mathrm{~h}$, after which the mixture was quenched with satd $\mathrm{NH}_{4} \mathrm{Cl}$ at $0{ }^{\circ} \mathrm{C}$ and extracted with $\mathrm{CH}_{2} \mathrm{Cl}_{2}$. The organic layers were washed with brine and dried over $\mathrm{MgSO}_{4}$. After being filtered and concentrated under vacuum, the crude product was used in the next step as obtained. The crude amine and $\mathrm{Boc}_{2} \mathrm{O}(21.8 \mathrm{mg}, 0.1 \mathrm{mmol}, 1$ equiv) were placed in a $10 \mathrm{~mL}$ round bottomed flask, and they were dissolved in $\mathrm{CH}_{2} \mathrm{Cl}_{2}(1 \mathrm{~mL})$. Next, $\mathrm{Et}_{3} \mathrm{~N}(14 \mu \mathrm{L}, 0.1 \mathrm{mmol}, 1$ equiv) was added. The mixture was stirred overnight and after that was purified by flash chromatography on silica gel using hexane/EtOAc mixtures (90:10 to 80:20) to afford the product $6 \mathrm{a}(24.7 \mathrm{mg}, 0.070 \mathrm{mmol})$ in $70 \%$ yield.

Enantiomeric excess (97\%) was determined by chiral HPLC (Chiralcel OD-H), hexane $-{ }^{i} \mathrm{PrOH}$ 90:10, $1.0 \mathrm{~mL} / \mathrm{min}$, major enantiomer $t_{\mathrm{r}}=40.96 \mathrm{~min}$, minor enantiomer $t_{\mathrm{r}}=51.38 \mathrm{~min}$. Brown oil; $[\alpha]_{\mathrm{D}}^{20}-9.77\left(c=1.37, \mathrm{CHCl}_{3}\right)(97 \% \mathrm{ee}) ;{ }^{1} \mathrm{H}$ NMR $(300 \mathrm{MHz}$, $\left.\mathrm{CD}_{3} \mathrm{OD}\right) \delta 7.43(\mathrm{~d}, J=7.5 \mathrm{~Hz}, 2 \mathrm{H}), 7.23(\mathrm{t}, J=7.5 \mathrm{~Hz}, 2 \mathrm{H}), 7.19-$ $7.07(\mathrm{~m}, 3 \mathrm{H}), 6.75(\mathrm{~d}, J=8.6 \mathrm{~Hz}, 1 \mathrm{H}), 6.31(\mathrm{~d}, J=3.1 \mathrm{~Hz}, 1 \mathrm{H}), 4.92$ $(\mathrm{m}, 1 \mathrm{H}), 4.27-4.04(\mathrm{~m}, 1 \mathrm{H}), 3.87(\mathrm{dd}, J=13.3,8.7 \mathrm{~Hz}, 1 \mathrm{H}), 1.39(\mathrm{~s}$, 9H); ${ }^{13} \mathrm{C}$ NMR (75 MHz, CD $\left.\mathrm{OD}\right) \delta 159.3$ (C), 149.9 (C), 145.3 (C), $133.7(\mathrm{C}), 130.7(\mathrm{C}), 130.2(\mathrm{CH}), 129.8(\mathrm{CH}), 127.6(\mathrm{CH})$, $126.5(\mathrm{CH}), 119.7(\mathrm{C}), 114.1(\mathrm{CH}), 112.1(\mathrm{CH}), 102.1(\mathrm{CH}), 80.8$ (C), $46.4(\mathrm{CH}), 45.5\left(\mathrm{CH}_{2}\right), 29.6\left(\mathrm{CH}_{3}\right)$; HRMS (ESI) $\mathrm{m} / z$ : $[\mathrm{M}+$ $\mathrm{H}]^{+}$calcd for $\mathrm{C}_{21} \mathrm{H}_{25} \mathrm{~N}_{2} \mathrm{O}_{3}, 353.1865$; found, 353.1862.

tert-Butyl (S)-(2-(6-Hydroxy-1H-indol-7-yl)-2-(4-(trifluoromethyl)phenyl)ethyl) Carbamate (6b). Compound $3 \mathrm{cc}(24.5 \mathrm{mg}, 0.07$ $\mathrm{mmol})$ and $\mathrm{NiCl}_{2} \cdot 6 \mathrm{H}_{2} \mathrm{O}(17.0 \mathrm{mg}, 0.07 \mathrm{mmol}, 1$ equiv) were placed in a $10 \mathrm{~mL}$ round bottomed flask, and $\mathrm{MeOH}(1 \mathrm{~mL})$ was added. After the suspension was stirred, $\mathrm{NaBH}_{4}(13.2 \mathrm{mg}, 0.35 \mathrm{mmol}, 5$ equiv) was added at $0{ }^{\circ} \mathrm{C}$, and the mixture was stirred at room temperature for 1.5 
h, after which the mixture was quenched with satd $\mathrm{NH}_{4} \mathrm{Cl}$ at $0{ }^{\circ} \mathrm{C}$ and extracted with $\mathrm{CH}_{2} \mathrm{Cl}_{2}$. The organic layers were washed with brine and dried over $\mathrm{MgSO}_{4}$. After being filtered and concentrated under vacuum, the crude product was used in the next step as obtained. The crude amine and $\mathrm{Boc}_{2} \mathrm{O}$ (15.3 mg, $0.07 \mathrm{mmol}, 1$ equiv) were placed in a $10 \mathrm{~mL}$ round bottomed flask, and they were disoslved in $\mathrm{CH}_{2} \mathrm{Cl}_{2}$ (1 $\mathrm{mL})$. Next, $\mathrm{Et}_{3} \mathrm{~N}$ ( $10 \mu \mathrm{L}, 0.07 \mathrm{mmol}, 1$ equiv) was added. The mixture was stirred overnight and after that was purified by flash chromatography on silica gel using hexane/EtOAc mixtures (90:10 to $80: 20)$ to afford the product $6 \mathbf{b}(18.2 \mathrm{mg}, 0.043 \mathrm{mmol})$ in $62 \%$ yield.

Enantiomeric excess (88\%) was determined by chiral HPLC (Chiralpak AD-H), hexane- ${ }^{i} \mathrm{PrOH}$ 80:20, $1.0 \mathrm{~mL} / \mathrm{min}$, major enantiomer $t_{\mathrm{r}}=18.0 \mathrm{~min}$, minor enantiomer $t_{\mathrm{r}}=20.76 \mathrm{~min}$. Colorless oil; $[\alpha]_{\mathrm{D}}^{20}-36.7\left(c=0.56, \mathrm{CHCl}_{3}\right)(86 \%$ ee $) ;{ }^{1} \mathrm{H}$ NMR $(300 \mathrm{MHz}$, $\left.\mathrm{CDCl}_{3}\right) \delta 7.95(\mathrm{~s}, 1 \mathrm{H}), 7.50(\mathrm{~d}, J=8.3 \mathrm{~Hz}, 3 \mathrm{H}), 7.42(\mathrm{~d}, J=8.3 \mathrm{~Hz}$, $3 \mathrm{H}), 7.37(\mathrm{~d}, J=8.3 \mathrm{~Hz}, 1 \mathrm{H}), 6.93(\mathrm{t}, J=2.8 \mathrm{~Hz}, 1 \mathrm{H}), 6.67(\mathrm{~d}, J=8.4$ $\mathrm{Hz}, 1 \mathrm{H}), 6.41(\mathrm{dd}, J=3.3,1.9 \mathrm{~Hz}, 1 \mathrm{H}), 5.55(\mathrm{~s}, 1 \mathrm{H}), 5.00-4.83(\mathrm{~m}$, $2 \mathrm{H}), 4.04(\mathrm{~m}, 1 \mathrm{H}), 3.84(\mathrm{ddd}, J=13.7,8.4,5.4 \mathrm{~Hz}, 1 \mathrm{H}), 1.36(\mathrm{~s}$, $10 \mathrm{H}) ;{ }^{13} \mathrm{C}$ NMR (75 MHz, $\left.\mathrm{CDCl}_{3}\right) \delta 156.7$ (C), 149.7 (C), 145.2 (C), $136.0(\mathrm{C}), 129.0\left(\mathrm{q}, J_{\mathrm{C}-\mathrm{F}}=32 \mathrm{~Hz}, \mathrm{C}\right), 128.2(\mathrm{CH}), 125.7\left(\mathrm{q}, J_{\mathrm{C}-\mathrm{F}}\right.$ $=3.4 \mathrm{~Hz}, \mathrm{CH}), 124.1\left(\mathrm{q}, J_{\mathrm{C}-\mathrm{F}}=272.0 \mathrm{~Hz}, \mathrm{CF}_{3}\right), 123.5(\mathrm{CH}), 123.0$ (C), $120.4(\mathrm{CH}), 111.1(\mathrm{CH}), 109.1(\mathrm{C}), 102.8(\mathrm{CH}), 80.1(\mathrm{C}) 43.0$ $\left(\mathrm{CH}_{2}\right), 42.2(\mathrm{CH}), 28.3\left(\mathrm{CH}_{3}\right)$; HRMS (ESI) $\mathrm{m} / z$ : $[\mathrm{M}+\mathrm{H}]^{+}$calcd for $\mathrm{C}_{22} \mathrm{H}_{24} \mathrm{~F}_{3} \mathrm{~N}_{2} \mathrm{O}_{3}$, 421.1734; found, 421.1739.

Procedure and Characterization Data for Compound 7. Compound $3 \mathrm{ba}(28.1 \mathrm{mg}, 0.1 \mathrm{mmol})$ and 4-dimethylaminopyridine ( $36.7 \mathrm{mg}, 0.3 \mathrm{mmol}, 3$ equiv) were placed in a $10 \mathrm{~mL}$ round bottomed flask. Next, the flask was purged with $\mathrm{N}_{2}$, and $\mathrm{CH}_{2} \mathrm{Cl}_{2}(2 \mathrm{~mL})$ was added. After $5 \mathrm{~min}, \mathrm{~N}$-phenyl-bis(trifluoromethane sulfonimide (71.4 $\mathrm{mg}, 0.2 \mathrm{mmol}, 2$ equiv) was added, and the mixture was stirred at room temperature. The reaction was monitored by thin layer chromatography eluting with $\mathrm{CH}_{2} \mathrm{Cl}_{2}$. When the starting material was consumed, $\mathrm{H}_{2} \mathrm{O}$ was added $(5 \mathrm{~mL})$, and the mixture was extracted with EtOAc $(3 \times 20 \mathrm{~mL})$. The combined organic layers were washed with brine $(30 \mathrm{~mL})$ and dried under anhydrous $\mathrm{Na}_{2} \mathrm{SO}_{4}$. The organic solvents were removed under reduced pressure, and the residue was purified by column chromatography eluting with hexane: $\mathrm{CH}_{2} \mathrm{Cl}_{2}$ mixtures (50:50 to 30:70) to afford the triflated product $6(38.8 \mathrm{mg}$, $0.084 \mathrm{mmol})$ in $84 \%$ yield.

(S)-4-(2-Nitro-1-phenylethyl)-1H-indol-5-yl Trifluoromethanesulfonate (7). Enantiomeric excess (96\%) was determined by chiral HPLC (Chiralpak AD-H), hexane- ${ }^{i} \mathrm{PrOH} 80: 20,1.0 \mathrm{~mL} / \mathrm{min}$, major enantiomer $t_{\mathrm{r}}=7.19 \mathrm{~min}$, minor enantiomer $t_{\mathrm{r}}=8.19 \mathrm{~min}$. Brown oil; $[\alpha]_{\mathrm{D}}^{20}-55.71\left(c=0.53, \mathrm{CHCl}_{3}\right)(96 \%$ ee $) ;{ }^{1} \mathrm{H}$ NMR $(300 \mathrm{MHz}$, $\left.\mathrm{CDCl}_{3}\right) \delta 8.40(\mathrm{bs}, 1 \mathrm{H}), 7.37-7.23(\mathrm{~m}, 7 \mathrm{H}), 7.23-7.20(\mathrm{~m}, 1 \mathrm{H}), 7.16$ $(\mathrm{d}, J=8.9 \mathrm{~Hz}, 1 \mathrm{H}), 6.34(\mathrm{ddd}, J=3.2,2.0,0.9 \mathrm{~Hz}, 1 \mathrm{H}), 5.69(\mathrm{dd}, J=$ $8.1,7.3 \mathrm{~Hz}, 1 \mathrm{H}), 5.43(\mathrm{dd}, J=13.4,8.4 \mathrm{~Hz}, 1 \mathrm{H}), 5.12(\mathrm{dd}, J=13.4,7.0$ $\mathrm{Hz}, 1 \mathrm{H}) ;{ }^{13} \mathrm{C}$ NMR $\left(75.5 \mathrm{MHz}, \mathrm{CDCl}_{3}\right) \delta 141.1$ (C), 137.8 (C), 135.0 (C), $128.9(\mathrm{CH}), 127.4(\mathrm{CH}), 127.3(\mathrm{CH}), 127.0(\mathrm{C}), 126.9(\mathrm{CH})$, $123.6(\mathrm{C}), 118.6\left(\mathrm{q}, J_{\mathrm{C}-\mathrm{F}}=320.2 \mathrm{~Hz}, \mathrm{C}\right), 115.6(\mathrm{CH}), 112.1(\mathrm{CH})$, $102.5(\mathrm{CH}), 77.0\left(\mathrm{CH}_{2}\right), 41.3(\mathrm{CH}) ;{ }^{19} \mathrm{~F}$ NMR $\left(282 \mathrm{MHz}, \mathrm{CDCl}_{3}\right) \delta$ -74.16 (s); HRMS (ESI) $m / z$ : $[\mathrm{M}+\mathrm{H}]^{+}$calcd for $\mathrm{C}_{17} \mathrm{H}_{14} \mathrm{~F}_{3} \mathrm{~N}_{2} \mathrm{O}_{5} \mathrm{~S}$, 415.0570; found, 415.0577

Procedure and Characterization Data for Compound 8. Compound $7(22.4 \mathrm{mg}, 0.054 \mathrm{mmol})$ was placed in a $25 \mathrm{~mL}$ round bottomed flask, which was purged with $\mathrm{N}_{2}$. Next, $\mathrm{MeOH}(3 \mathrm{~mL})$ was added, followed by $10 \% \mathrm{Pd} / \mathrm{C}(5 \mathrm{mg})$ and $\mathrm{Et}_{2} \mathrm{NH}(6.7 \mu \mathrm{L}, 0.065$ mmol, 1.2 equiv). The reaction vessel then was repeatedly purged with $\mathrm{H}_{2}$ using a balloon and a needle as a vent. Finally, the reaction was stirred at room temperature under $\mathrm{H}_{2}$ ( $1 \mathrm{~atm}$, balloon). The reaction was monitored by thin layer chromatography eluting with $\mathrm{CH}_{2} \mathrm{Cl}_{2}$. When the reaction was complete, the suspension was passed through a pad of Celite, and the organic solvents were removed under vacuum. The residue then was placed in a $10 \mathrm{~mL}$ round bottomed flask, which was purged with $\mathrm{N}_{2} \cdot \mathrm{CH}_{2} \mathrm{Cl}_{2}$ was added $(1.5 \mathrm{~mL})$, and, when it was dissolved, $\mathrm{AcCl}\left(5.8 \mu \mathrm{L}, 0.081 \mathrm{mmol}, 1.5\right.$ equiv) and $\mathrm{Et}_{3} \mathrm{~N}(12.0 \mu \mathrm{L}$, $0.086 \mathrm{mmol}, 1.6$ equiv) were carefully added via syringe and the mixture was stirred at room temperature until completion (TLC). The reaction mixture was quenched by addition of $\mathrm{H}_{2} \mathrm{O}$ at $0{ }^{\circ} \mathrm{C}$ and extracted three times with $\mathrm{CH}_{2} \mathrm{Cl}_{2}$. The combined organic layers were washed with brine, dried over anhydrous $\mathrm{MgSO}_{4}$, and the filtrate was concentrated to dryness in vacuo. The crude was purified by column chromatography eluting with hexane:AcOEt mixtures (95:5 to 80:20) to obtain the product $8(11.3 \mathrm{mg}, 0.041 \mathrm{mmol})$ in $75 \%$ yield.

(S)-N-(2-(1H-Indol-4-yl)-2-phenylethyl)acetamide (8). Enantiomeric excess $(93 \%)$ was determined by chiral HPLC (Chiralcel OD$\mathrm{H})$, hexane $-{ }^{i} \mathrm{PrOH} 85: 15,1.0 \mathrm{~mL} / \mathrm{min}$, major enantiomer $t_{\mathrm{r}}=35.04$ min, minor enantiomer $t_{\mathrm{r}}=38.99 \mathrm{~min}$. Transparent oil; $[\alpha]_{\mathrm{D}}^{20}-16.73$ $\left(c=0.53, \mathrm{CHCl}_{3}\right)(93 \% \mathrm{ee}) ;{ }^{1} \mathrm{H} \mathrm{NMR}\left(300 \mathrm{MHz}, \mathrm{CDCl}_{3}\right) \delta 8.32(\mathrm{~s}$, $1 \mathrm{H}), 7.35-7.27(\mathrm{~m}, 5 \mathrm{H}), 7.24-7.14(\mathrm{~m}, 3 \mathrm{H}), 6.99(\mathrm{~d}, J=7.2 \mathrm{~Hz}$, $1 \mathrm{H}), 6.57$ (ddd, $J=3.1,2.0,0.9 \mathrm{~Hz}, 1 \mathrm{H}), 5.48(\mathrm{~s}, 1 \mathrm{H}), 4.62(\mathrm{t}, J=7.9$ $\mathrm{Hz}, 1 \mathrm{H}), 4.10-3.96(\mathrm{~m}, 2 \mathrm{H}), 1.86(\mathrm{~s}, 3 \mathrm{H}) ;{ }^{13} \mathrm{C} \mathrm{NMR}(75.5 \mathrm{MHz}$, $\left.\mathrm{CDCl}_{3}\right) \delta 170.1(\mathrm{C}), 142.0(\mathrm{C}), 136.0(\mathrm{C}), 135.0(\mathrm{C}), 128.6(\mathrm{CH})$, $128.1(\mathrm{CH}), 127.5(\mathrm{C}), 126.6(\mathrm{CH}), 124.1(\mathrm{CH}), 122.1(\mathrm{CH}), 117.7$ $(\mathrm{CH}), 110.0(\mathrm{CH}), 101.1(\mathrm{CH}), 47.9(\mathrm{CH}), 43.5\left(\mathrm{CH}_{2}\right), 23.4\left(\mathrm{CH}_{3}\right)$; HRMS (ESI) $m / z:[\mathrm{M}+\mathrm{H}]^{+}$calcd for $\mathrm{C}_{18} \mathrm{H}_{19} \mathrm{~N}_{2} \mathrm{O}, 279.1492$; found, 279.1491.

Procedure and Characterization Data for Compound 9. Compound 3ba $(28.1 \mathrm{mg}, 0.1 \mathrm{mmol})$ and racemic $1,1^{\prime}$-binaphthyl2,2'-diyl hydrogen phosphate $(2.8 \mathrm{mg}, 0.01 \mathrm{mmol}, 0.1$ equiv) were placed in a $10 \mathrm{~mL}$ flask. The flask then was purged with $\mathrm{N}_{2}$, and $\mathrm{CH}_{2} \mathrm{Cl}_{2}(1 \mathrm{~mL})$ was added. After being stirred for $10 \mathrm{~min}$, methylvinylketone $(9.8 \mu \mathrm{L}, 0.12 \mathrm{mmol}, 1.2$ equiv) was added via syringe. The mixture was stirred at room temperature for $24 \mathrm{~h}$. Next, $\mathrm{H}_{2} \mathrm{O}(5 \mathrm{~mL})$ was added and the mixture was extracted with $\mathrm{CH}_{2} \mathrm{Cl}_{2}(3$ $\times 20 \mathrm{~mL}$ ). The combined organic layers were washed with brine and dried over $\mathrm{Na}_{2} \mathrm{SO}_{4}$. Finally, the residue was purified by column chromatography eluting with hexane:AcOEt mixtures (95:5 to 75:25), and the C-3 alkylated product $9(27.5 \mathrm{mg}, 0.078 \mathrm{mmol})$ was obtained in $78 \%$ yield.

(S)-4-(5-Hydroxy-4-(2-nitro-1-phenylethyl)-1H-indol-3-yl)butan2-one (9). Enantiomeric excess (96\%) was determined by chiral HPLC (Chiralpak AS-H), hexane- ${ }^{i} \mathrm{PrOH}$ 80:20, $1.0 \mathrm{~mL} / \mathrm{min}$, major enantiomer $t_{\mathrm{r}}=19.52 \mathrm{~min}$, minor enantiomer $t_{\mathrm{r}}=24.65 \mathrm{~min}$. Colorless oil; $[\alpha]_{\mathrm{D}}^{20}-40.88\left(c=1.07, \mathrm{CHCl}_{3}\right)(96 \%$ ee $) ;{ }^{1} \mathrm{H}$ NMR $\left(300 \mathrm{MHz}, \mathrm{CDCl}_{3}\right) \delta 7.96(\mathrm{~s}, 1 \mathrm{H}), 7.34-7.19(\mathrm{~m}, 5 \mathrm{H}), 7.13(\mathrm{~d}, J=$ $8.5 \mathrm{~Hz}, 1 \mathrm{H}), 6.94(\mathrm{~d}, J=2.0 \mathrm{~Hz}, 1 \mathrm{H}), 6.65(\mathrm{~d}, J=8.6 \mathrm{~Hz}, 1 \mathrm{H}), 5.73(\mathrm{t}$, $J=7.4 \mathrm{~Hz}, 1 \mathrm{H}), 5.46(\mathrm{dd}, J=12.9,7.5 \mathrm{~Hz}, 1 \mathrm{H}), 5.31(\mathrm{dd}, J=12.9,7.3$ $\mathrm{Hz}, 1 \mathrm{H}), 4.84(\mathrm{~s}, 1 \mathrm{H}), 3.24(\mathrm{td}, J=7.6,3.9 \mathrm{~Hz}, 2 \mathrm{H}), 2.96-2.66(\mathrm{~m}$, $2 \mathrm{H}), 2.08(\mathrm{~s}, 3 \mathrm{H}) ;{ }^{13} \mathrm{C} \mathrm{NMR}\left(75 \mathrm{MHz}, \mathrm{CDCl}_{3}\right) \delta 208.9(\mathrm{C}), 147.7$ (C), $139.7(\mathrm{C}), 132.7(\mathrm{C}), 128.7(\mathrm{CH}), 127.5(\mathrm{CH}), 127.0(\mathrm{CH})$, $126.4(\mathrm{C}), 124.3(\mathrm{CH}), 116.2(\mathrm{C}), 114.9(\mathrm{C}), 113.3(\mathrm{CH}), 111.5$ $(\mathrm{CH}), 78.7\left(\mathrm{CH}_{2}\right), 44.6\left(\mathrm{CH}_{2}\right), 42.3(\mathrm{CH}), 29.8\left(\mathrm{CH}_{3}\right), 21.8\left(\mathrm{CH}_{2}\right)$; HRMS (ESI) $m / z:[\mathrm{M}+\mathrm{Na}]^{+}$calcd for $\mathrm{C}_{20} \mathrm{H}_{20} \mathrm{~N}_{2} \mathrm{O}_{4} \mathrm{Na}$, 375.1315; found, 375.1322 .

Control Experiment with 5-Methoxyindole. In a $5 \mathrm{~mL}$ vial were placed 5 -methoxyindole $(0.15 \mathrm{mmol})$, trans- $\beta$-nitrostyrene $2 \mathrm{a}$ $(0.10 \mathrm{mmol})$, and Rawal squaramide (VI, $0.84 \mathrm{mg}, 0.02 \mathrm{mmol})$. The mixture then was dissolved in $\mathrm{CHCl}_{3}(1 \mathrm{~mL})$, and it was stirred at 4 ${ }^{\circ} \mathrm{C}$ until the reaction was complete (TLC). Finally, the reaction mixture was directly poured into the column chromatography using hexane:AcOEt mixtures (90:10 to 80:20) as eluent to afford the product 10 as an enantioenriched mixture.

5-Methoxy-3-(1-phenyl-2-nitroethyl)-1H-indole (10). The product 10 was obtained as a colorless oil $(3 \mathrm{mg}, 0.007 \mathrm{mmol}, 7 \%$ yield $)$. Enantiomeric excess (14\%) was determined by chiral HPLC (Chiralpak AD-H), hexane- ${ }^{i} \mathrm{PrOH}$ 90:10, $1.0 \mathrm{~mL} / \mathrm{min}$, major enantiomer $t_{\mathrm{r}}=29.92 \mathrm{~min}$, minor enantiomer $t_{\mathrm{r}}=44.34 \mathrm{~min}$. The HPLC conditions were previously described in the literature. ${ }^{30}{ }^{1} \mathrm{H}$ NMR $\left(300 \mathrm{MHz}, \mathrm{CDCl}_{3}\right) \delta 7.97(\mathrm{~s}, 1 \mathrm{H}), 7.33-7.24(\mathrm{~m}, 5 \mathrm{H}), 7.22(\mathrm{~d}$, $J=1.0 \mathrm{~Hz}, 1 \mathrm{H}), 7.01-6.99(\mathrm{~m}, 1 \mathrm{H}), 6.86-6.80(\mathrm{~m}, 2 \mathrm{H}), 5.12(\mathrm{t}, J=$ $7.5 \mathrm{~Hz}, 1 \mathrm{H}), 5.03(\mathrm{dd}, J=12.2,7.4 \mathrm{~Hz}, 1 \mathrm{H}), 4.92(\mathrm{dd}, J=12.2,8.4 \mathrm{~Hz}$, $1 \mathrm{H}), 3.75(\mathrm{~s}, 3 \mathrm{H}) ;{ }^{13} \mathrm{C}$ NMR $\left(75 \mathrm{MHz}, \mathrm{CDCl}_{3}\right) \delta 154.2(\mathrm{C}), 139.1$ (C), $131.6(\mathrm{C}), 128.9(\mathrm{CH}), 127.8(\mathrm{CH}), 127.6(\mathrm{CH}), 126.6(\mathrm{C})$, $122.3(\mathrm{CH}), 114.2(\mathrm{C}), 112.8(\mathrm{CH}), 112.1(\mathrm{CH}), 100.9(\mathrm{CH}), 79.5$ $\left(\mathrm{CH}_{2}\right), 55.8\left(\mathrm{CH}_{3}\right), 41.5(\mathrm{CH})$. 


\section{ASSOCIATED CONTENT}

\section{S Supporting Information}

The Supporting Information is available free of charge on the ACS Publications website at DOI: 10.1021/acs.joc.8b00612.

${ }^{1} \mathrm{H}$ and ${ }^{13} \mathrm{C}$ NMR spectra, and HPLC chromatograms for all compounds (PDF)

Crystallographic data for compound $3 \mathrm{ab}$ (CIF)

Crystallographic data for compound $3 \mathrm{eb}$ (CIF)

\section{AUTHOR INFORMATION}

\section{Corresponding Authors}

*E-mail: carlos.vila@uv.es.

*E-mail: jose.r.pedro@uv.es.

ORCID $\odot$

Carlos Vila: 0000-0001-9306-1109

Gonzalo Blay: 0000-0002-7379-6789

José R. Pedro: 0000-0002-6137-866X

\section{Author Contributions}

§.R.-B. and R.S.-G. contributed equally.

\section{Notes}

The authors declare no competing financial interest.

\section{ACKNOWLEDGMENTS}

Financial support from the MINECO (Gobierno de España; CTQ2017-84900-P) is gratefully acknowledged. C.V. thanks MINECO for a JdC contract. J.R.-B. thanks the Ministry of Education for a Collaboration grant. Access to NMR, MS, and X-ray facilities from the Servei Central de Suport a la Investigació Experimental (SCSIE)-UV is also acknowledged.

\section{REFERENCES}

(1) (a) The Biochemical Basis of Neuropharmacology, 8th ed.; Cooper, J. R., Bloom, F. E., Roth, R. H., Eds.; Oxford University Press, Inc: New York, 2003. For arylethylamines bearing an indole group, see: (b) Bernotas, R. C.; Coghlan, R. D.; Heffernan, G. D.; Li, Y.; Mcdevitt, R. E.; Robichaud, A. J. Sulfonyl substituted $1 H$-indoles as ligands for the 5-hydroxytryptamine receptors. Patent WO 2007084841 A3, 2007. (c) Cassady, J. M.; Clemens, J. A. Use of 4(2-di- $\mathrm{N}$-propylaminoethyl)indole or a salt thereof as a presynaptic dopamine autoreceptor stimulant. Patent US 4378368 A, 1983. (d) Mehndiratta, S.; Wang, R.-S.; Huang, H.-L.; Su, C.-J.; Hsu, C.-M.; Wu, Y.-W.; Pan, S.-L.; Liou, J.-P. 4-Indolyl- $N$-hydroxyphenylacrylamides as potent HDAC class I and IIB inhibitors in vitro and in vivo. Eur. J. Med. Chem. 2017, 134, 13-23.

(2) (a) Zhang, A.; Neumeyer, J. L.; Baldessarini, R. J. Recent Progress in Development of Dopamine Receptor Subtype-Selective Agents: Potential Therapeutics for Neurological and Psychiatric Disorders. Chem. Rev. 2007, 107, 274-302. (b) Zhang, J.; Xiong, B.; Zhen, X.; Zhang, A. Dopamine D1 receptor ligands: where are we now and where are we going. Med. Res. Rev. 2009, 29, 272-294.

(3) (a) Indoles; Sundberg, R. J., Ed.; Academic Press: San Diego, CA, 1996. (b) Bronner, S. M.; Im, G. Y. J.; Garg, N. K. Heterocycles in Natural Product Synthesis; Wiley-VCH Verlag GmbH \& Co. KGaA: New York, 2011; pp 221-265.

(4) (a) Kaushik, N. K.; Kaushik, N.; Attri, P.; Kumar, N.; Kim, C. H.; Verma, A. K.; Choi, E. H. Biomedical Importance of Indoles. Molecules 2013, 18, 6620-6662. (b) Zhang, M. Z.; Chen, Q.; Yang, G.-F. A review on recent developments of indole-containing antiviral agents. Eur. J. Med. Chem. 2015, 89, 421-441. (c) Chadha, N.; Silakari, O. Indoles as therapeutics of interest in medicinal chemistry: Bird's eye view. Eur. J. Med. Chem. 2017, 134, 159-184.

(5) Barden, T. C. Topics in Heterocyclic Chemistry; Springer-Verlag: Berlin, 2010; pp 31-46.
(6) Manickam, M.; Iqbal, P.; Belloni, M.; Kumar, S.; Preece, J. A. A Brief Review of Carbazole-Based Photorefractive Liquid Crystalline Materials. Isr. J. Chem. 2012, 52, 917-934.

(7) (a) Comprehensive Asymmetric Catalysis; Jacobsen, E. N., Pfaltz, A., Yamamoto, H., Eds.; Springer: New York, 1999. (b) New Frontiers in Asymmetric Catalysis; Mikami, K., Lautens, M., Eds.; Wiley-WCH: New York, 2007.

(8) (a) Bandini, M.; Eichholzer, A. Catalytic Functionalization of Indoles in a New Dimension. Angew. Chem., Int. Ed. 2009, 48, 96089644. (b) Bartoli, G.; Bencivenni, G.; Dalpozzo, R. Organocatalytic strategies for the asymmetric functionalization of indoles. Chem. Soc. Rev. 2010, 39, 4449-4465. (c) Marques-Lopez, E.; Diez-Martinez, A.; Merino, P.; Herrera, R. P. The role of the indole in important organocatalytic enantioselective Friedel-Crafts alkylation reactions. Curr. Org. Chem. 2009, 13, 1585-1609.

(9) (a) Alonso, D. A.; Baeza, A.; Chinchilla, R.; Gómez, C.; Guillena, G.; Pastor, I. M.; Ramón, D. J. Recent advances in asymmetric organocatalyzed conjugate additions to nitroalkenes. Molecules 2017, 22, 895-946. (b) Berner, O. M.; Tedeschi, L.; Enders, D. Asymmetric Michael additions to nitroalkenes. Eur. J. Org. Chem. 2002, 2002, $1877-1894$.

(10) Barrett, A. G. M.; Graboski, G. G. Conjugated nitroalkenes: versatile intermediates in organic synthesis. Chem. Rev. 1986, 86, 751762.

(11) For selected examples of enantioselective functionalization at C2, see: (a) Taylor, M. S.; Jacobsen, E. N. Highly enantioselective catalytic acyl-Pictet-Spengler reactions. J. Am. Chem. Soc. 2004, 126, 10558-10559. (b) Evans, D. A.; Fandrick, K. R.; Song, H.-J.; Scheidt, K. A.; Xu, R. Enantioselective Friedel-Crafts alkylations catalyzed by bis(oxazolinyl)pyridine-Scandium(III) triflate complexes. J. Am. Chem. Soc. 2007, 129, 10029-10041. (c) Blay, G.; Fernández, I.; Pedro, J. R.; Vila, C. Catalytic enantioselective Friedel-Crafts alkylation at the 2-position of indole with simple enones. Tetrahedron Lett. 2007, 48, 6731-6734. (d) Zeng, M.; Kang, Q.; He, Q.-L.; You, S.L. Highly enantioselective Friedel-Crafts reaction of 4,7-dihydroindoles with $\beta, \gamma$-unsaturated $\alpha$-keto esters by chiral Brønsted acids. Adv. Synth. Catal. 2008, 350, 2169-2173. (e) Hong, L.; Sun, W.; Liu, C.; Wang, L.; Wong, K.; Wang, R. Enantioselective Friedel-Crafts alkylation of 4,7-dihydroindoles with enones catalyzed by primarysecondary diamines. Chem. - Eur. J. 2009, 15, 11105-11108. (f) Cheng, H.-G.; Lu, L.-Q.; Wang, T.; Yang, Q.-Q.; Liu, X.-P.; Li, Y.; Deng, Q.-H.; Chen, J.-R.; Xiao, W.-J. Highly enantioselective Friedel-Crafts alkylation/ $\mathrm{N}$-hemiacetalization cascade reaction with indoles. Angew. Chem., Int. Ed. 2013, 52, 3250-3254.

(12) For selected examples of enantioselective functionalization at the N-1, see: (a) Hong, L.; Sun, W.; Liu, C.; Wang, L.; Wang, R. Asymmetric organocatalytic $\mathrm{N}$-alkylation of indole-2-carbaldehydes with $\alpha, \beta$-unsaturated aldehydes: one-pot synthesis of chiral pyrrolo[1,2-a]indole-2-carbaldehydes. Chem. - Eur. J. 2010, 16, 440-444. (b) Bandini, M.; Eichholzer, A.; Tragni, M.; Umani-Ronchi, A. Enantioselective phase-transfer-catalyzed intramolecular Aza-Michael reaction: effective route to pyrazino-indole compounds. Angew. Chem., Int. Ed. 2008, 47, 3238-3241. (c) Cui, H.-L.; Feng, X.; Peng, J.; Lei, J.; Jiang, K.; Chen, Y.-C. Chemoselective asymmetric $N$-allylic alkylation of indoles with Morita-Baylis-Hillman carbonates. Angew. Chem., Int. Ed. 2009, 48, 5737-5740. (d) Stanley, L. M.; Hartwig, J. F. Iridiumcatalyzed regio- and enantioselective $\mathrm{N}$-allylation of indoles. Angew. Chem., Int. Ed. 2009, 48, 7841-7844. (e) Enders, D.; Greb, A.; Deckers, K.; Selig, P.; Merkens, C. Quadruple domino organocatalysis: an asymmetric Aza-Michael/Michael/Michael/Aldol reaction sequence leading to tetracyclic indole structures with six stereocenters. Chem. - Eur. J. 2012, 18, 10226-10229.

(13) (a) Sandtorv, A. H. Transition metal-catalyzed C-H activation of indoles. Adv. Synth. Catal. 2015, 357, 2403-2435. (b) Leitch, J. A.; Bhonoah, Y.; Frost, C. G. Beyond C2 and C3: transition-metalcatalyzed C-H functionalization of indole. ACS Catal. 2017, 7, 56185627.

(14) (a) Montesinos-Magraner, M.; Vila, C.; Rendón-Patiño, A.; Blay, G.; Fernández, I.; Muñoz, M. C.; Pedro, J. R. Organocatalytic 
enantioselective Friedel-Crafts aminoalkylation of indoles in the carbocyclic ring. ACS Catal. 2016, 6, 2689-2693. (b) MontesinosMagraner, M.; Vila, C.; Blay, G.; Fernández, I.; Muñoz, M. C.; Pedro, J. R. Hydroxy-directed enantioselective hydroxyalkylation in the carbocyclic ring of indoles. Org. Lett. 2017, 19, 1546-1549.

(15) Jørgensen has described just one example of Friedel-Crafts/ oxa-Michael reaction of 4-hydroxyindole occurring at the C-5 position of the carbocyclic ring: Poulsen, P. H.; Feu, K. S.; Paz, B. M.; Jensen, F.; Jørgensen, K. A. Organocatalytic asymmetric 1,6-addition/1,4addition sequence to 2,4-dienals for the synthesis of chiral chromans. Angew. Chem., Int. Ed. 2015, 54, 8203-8207.

(16) For the earlier nonenantioselective examples of the activating/ directing ability of the $\mathrm{OH}$ group in hydroxyindoles, see: (a) Monti, S. A.; Johnson, W. O.; White, D. H. The Mannich reaction of hydroxyindoles. Tetrahedron Lett. 1966, 7, 4459-4464. (b) Troxler, F.; Bormann, G.; Seemann, F. Synthesen von Mannich-Basen von Hydroxy-indolen. 3. Mitteilung über synthetische Indol-Verbindungen. Helv. Chim. Acta 1968, 51, 1203-1213.

(17) For a review, see: (a) Chunchao, W.; Shaolei, X.; Zhengfeng, X. Research progress in Friedel-Crafts reaction of indoles and nitroalkenes. Chin. J. Org. Chem. 2013, 33, 1919-1931. For a review of 3-(2-nitroalkyl)indoles, see: (b) Lancianesi, S.; Palmieri, A.; Petrini, M. Synthetic approaches to 3-(2-nitroalkyl) indoles and their use to access tryptamines and related bioactive compounds. Chem. Rev. 2014, 114, 7108-7149.

(18) For selected examples at C-3, see: (a) Herrera, R. P.; Sgarzani, V.; Bernardi, L.; Ricci, A. Catalytic enantioselective Friedel-Crafts alkylation of indoles with nitroalkenes by using a simple thiourea organocatalyst. Angew. Chem., Int. Ed. 2005, 44, 6576-6579. (b) Jia, Y.-X.; Zhu, S.-F.; Yang, Y.; Zhou, Q.-L. Asymmetric Friedel-Crafts alkylations of indoles with nitroalkenes catalyzed by $\mathrm{Zn}(\mathrm{II})-$ bisoxazoline complexes. J. Org. Chem. 2006, 71, 75-80. (c) Lu, S.F.; Du, D.-M.; Xu, J. Enantioselective Friedel-Crafts alkylation of indoles with nitroalkenes catalyzed by bifunctional tridentate bis(oxazoline) $-\mathrm{Zn}$ (II) complex. Org. Lett. 2006, 8, 2115-2118. (d) Itoh, J.; Fuchibe, K.; Akiyama, T. Chiral phosphoric acid catalyzed enantioselective Friedel-Crafts alkylation of indoles with nitroalkenes: cooperative effect of $3 \AA$ molecular sieves. Angew. Chem., Int. Ed. 2008, 47, 4016-4018. (e) Guo, F.; Lai, G.; Xiong, S.; Wang, S.; Wang, Z. Monodentate $N$-ligand-directed bifunctional transition-metal catalysis: highly enantioselective Friedel-Crafts alkylation of indoles with nitroalkenes. Chem. - Eur. J. 2010, 16, 6438-6441. (f) Gao, J. R.; Wu, H.; Xiang, B.; Yu, W.-B.; Han, L.; Jia, Y.-X. Highly enantioselective construction of trifluoromethylated all-carbon quaternary stereocenters via Nickel-catalyzed Friedel-Crafts alkylation reaction. J. Am. Chem. Soc. 2013, 135, 2983-2986 For selected examples at C-2, see:. (g) Sheng, Y.-F.; Li, G.-Q.; Kang, Q.; Zhang, A.J.; You, S.-L. Asymmetric Friedel-Crafts reaction of 4,7-dihydroindoles with nitroolefins by chiral Brønsted acids under low catalyst loading. Chem. - Eur. J. 2009, 15, 3351-3354. (h) Takenaka, N.; Chen, J.; Captain, B.; Sarangthem, R. S.; Chandrakuma, A. Helical chiral 2aminopyridinium ions: a new class of hydrogen bond donor catalysts. J. Am. Chem. Soc. 2010, 132, 4536-4537. (i) Weng, J.-Q.; Fan, R.-J.; Deng, Q.-M.; Liu, R.-R.; Gao, J.-R.; Jia, Y.-X. Enantioselective FriedelCrafts alkylation reactions of 3 -substituted indoles with electrondeficient alkenes. J. Org. Chem. 2016, 81, 3023-3030.

(19) (a) Tian, S.-K.; Chen, Y.-G.; Hang, J. F.; Tang, L.; McDaid, P.; Deng, L. Asymmetric organic catalysis with modified cinchona alkaloids. Acc. Chem. Res. 2004, 37, 621-631. (b) Takemoto, Y. Recognition and activation by ureas and thioureas: stereoselective reactions using ureas and thioureas as hydrogen-bonding donors. Org. Biomol. Chem. 2005, 3, 4299-4306. (c) Doyle, A. G.; Jacobsen, E. N. Small-molecule H-bond donors in asymmetric catalysis. Chem. Rev. 2007, 107, 5713-5743. (d) Aleman, J.; Parra, A.; Jiang, H.; Jørgensen, K. A. Squaramides: bridging from molecular recognition to bifunctional organocatalysis. Chem. - Eur. J. 2011, 17, 6890-6899.

(20) Zhu, Y.; Malerich, J. P.; Rawal, V. H. Squaramide-catalyzed enantioselective Michael addition of diphenyl phosphite to nitroalkenes. Angew. Chem., Int. Ed. 2010, 49, 153-156.
(21) CCDC 1821099 (3ab) and CCDC 1821100 (3eb) contain the supplementary crystallographic data for this Article. These data can be obtained free of charge from The Cambridge Crystallographic Data Centre via www.ccdc.cam.ac.uk/data_request/cif.

(22) Chauhan, P.; Mahajan, S.; Kaya, U.; Hack, D.; Enders, D. Bifunctional amine-squaramides: powerful hydrogen-bonding organocatalysts for asymmetric domino/cascade reactions. Adv. Synth. Catal. 2015, 357, 253-281.

(23) (a) Stang, P. J.; Hanack, M.; Subramanian, L. R. Perfluoroalkanesulfonic esters: methods of preparation and applications in organic chemistry. Synthesis 1982, 1982, 85-126. (b) Ritter, K. Synthetic transformations of vinyl and aryl triflates. Synthesis 1993, 1993, 735-762.

(24) During the preparation of the manuscript, two examples were shown of enantioselective functionalization of the carbocyclic ring of indoles: (a) Xiao, M.; Xu, D.; Liang, W.; Wu, W. M.; Chan, A. S. C.; Zhao, J. Organocatalytic enantioselective Friedel-Crafts alkylation/ lactonization reaction of hydroxyindoles with methyleneoxindoles. Adv. Synth. Catal. 2018, 360, 917-924. (b) Xun, W.; Xu, B.; Chen, B.; Meng, S.; Chan, A. S. C.; Qiu, F. G.; Zhao, J. Regio and enantioselective organocatalytic Friedel-Crafts alkylation of 4-aminoindoles at the C7-position. Org. Lett. 2018, 20, 590-593.

(25) Li, H.; Wang, Y.; Tang, L.; Deng, L. Highly enantioselective conjugate addition of malonate and $\beta$-ketoester to nitroalkenes: asymmetric $\mathrm{C}-\mathrm{C}$ bond formation with new bifunctional organic catalysts based on cinchona alkaloids. J. Am. Chem. Soc. 2004, 126, 9906-9907.

(26) Vakulya, B.; Varga, S.; Csámpal, A.; Soós, T. Highly enantioselective conjugate addition of nitromethane to chalcones using bifunctional cinchona organocatalysts. Org. Lett. 2005, 7, 19671969.

(27) Yang, W.; Du, D.-M. Highly enantioselective michael addition of nitroalkanes to chalcones using chiral squaramides as hydrogen bonding organocatalysts. Org. Lett. 2010, 12, 5450-5453.

(28) Simpson, A. J.; Lam, H. W. Enantioselective nickel-catalyzed Michael additions of 2-acetylazaarenes to nitroalkenes. Org. Lett. 2013, $15,2586-2589$.

(29) Terasaka, T.; Zenkoh, T.; Hayashida, H.; Matsuda, H.; Sato, J.; Imamura, Y.; Nagata, H.; Seki, N.; Tenda, Y.; Tasaki, M.; Takeda, M.; Tabuchi, S.; Yasuda, M.; Tsubaki, K. Polycyclic acid compounds useful as crth2 antagonists and antiallergic agents. PCT Int. Appl. WO 2008072784 A1, 2008.

(30) Wu, J.; Li, X.; Wu, F.; Wan, B. A new type of bis(sulfonamide)diamine ligand for a $\mathrm{Cu}(\mathrm{OTf})_{2}$-catalyzed asymmetric Friedel-Crafts alkylation reaction of indoles with nitroalkenes. Org. Lett. 2011, 13, 4834-4837. 